\title{
Quadratic Tangles in Planar Algebras
}

\author{
Vaughan F.R. Jones *
}

October 23, 2018

\begin{abstract}
In planar algebras, we show how to project certain simple "quadratic" tangles onto the linear space spanned by "linear" and "constant" tangles. We obtain some corollaries about the principal graphs and annular structure of subfactors.
\end{abstract}

\section{Introduction}

A planar algebra $\mathcal{P}$ consists of vector spaces $P_{n}$ together with multilinear operations between them indexed by planar tangles-large discs with internal ("input") discs all connected up by non-intersecting curves called strings. Thus a planar algebra may be thought of as made up from generators $R_{i} \in P_{n}$ to which linear combinations of planar tangles may be appplied to obtain all elements of $\mathcal{P}$.

It was shown in 20] that a certain kind of planar algebra, called a subfactor planar algebra, is equivalent to the standard invariant of an extremal finite index subfactor. We define this notion carefully in section 2.3.1, after which the term "planar algebra" will mean subfactor planar algebra.

The simplest tangles are ones without input discs which can be called constant tangles. They are the analogue of the identity in an ordinary associative algebra and supply a quotient of the Temperley-Lieb (TL) algebra in any unital planar algebra. Tangles of the next level of complexity are the annular tangles first appearing in [10]. In the terminology of this paper they should be called linear tangles but the term linear is somewhat loaded so we will tend to call them annular. They form a category and planar algebras can be decomposed as a module over the corresponding algebroid. This was done in [14]. The outcome is a sequence of numbers which we will call the "annular multiplicity sequence" $\left(a_{n}\right)$ which are the difference between the dimension of $P_{n}$ and the dimension of the image of the annular algebroid applied to $P_{k}$ for $k<n$. These numbers are easily obtained from the dimensions of the $P_{n}$ by a change of variable in the generating function-see [14]. For a subfactor algebra

\footnotetext{
${ }^{*}$ Supported in part by NSF Grant DMS 0856316, the Marsden fund UOA520, and the Swiss National Science Foundation.
} 
we define the "supertransitivity" to be largest $n$ for which $a_{k}=0$ for $1 \leq k \leq n$. The reason for the terminology is explained in section 3 (see also section 4 of [7]).

In the present paper we begin the much more difficult task of studying tangles with two input discs which we call "quadratic tangles". They are not closed under any natural operation and one problem is to uncover their mathematical structure. It is easy enough to list all quadratic tangles. However there is no guarantee that the elements of $\mathcal{P}$ obtained from different tangles will be linearly independent. Indeed even for the TL tangles this may not be so and it is precisely this linear dependence that gives rise to the discrete spectrum of the index for subfactors and the existence of $S U(2)$ TQFT's ([12],[21]). Linear dependence of labelled tangles is most easily approached if the tangle below gives a positive definite sesquilinear form on $P_{n}$ for each $n$. This positivity is one of the axioms of a subfactor planar algebra. (The precise meaning of these pictures will be explained in the next section, in the meantime one may just think of the $a$ 's and $b$ 's as tensors with indices on the distinguished boundary points and joining boundary points by a string signifies contraction of the corresponding indices.)

Fig. 1.0.1. The inner product tangle on $P_{n}($ for $n=3)$

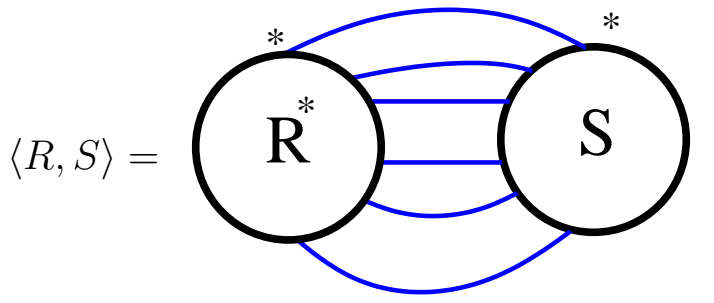

The dimension of the vector space spanned by a set of labelled tangles is then given by the rank of the matrix of inner products. In this way we were able to use the powerful results of [6] to obtain the dimensions of the relevant modules over the annular category in [14], [11]

Another frequently encountered tangle is the multiplication tangle:

Fig. 1.0.2.

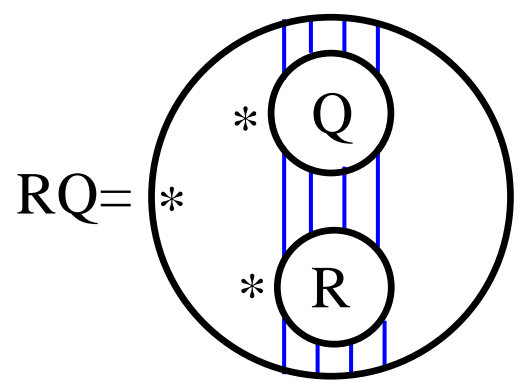

This tangle is particularly well understood. It turns each of the $P_{n}$ ( $n=4$ in the diagram) into an associative algebra which was the main ingredient in the first (obscure) appearance of subfactor PA's in [12].

In this paper we take a next step in the study of quadratic tangles by investigating the tangle $R \circ Q$ (and its rotations): 


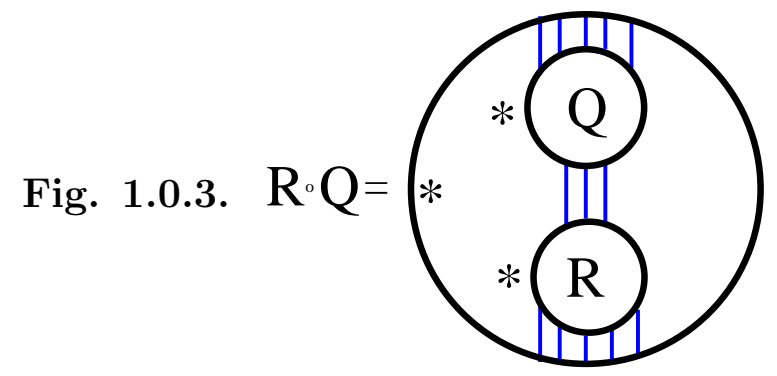

This tangle maps $P_{n} \otimes P_{n}$ to $P_{n+1}$ (again $n=4$ in the diagram). Its image may then be compared with the annular consequences of elements in $P_{k}$ for $k \leq n$. If $\mathcal{P}$ is $(n-1)$ supertransitive the only such annular consequences are the Temperley-Lieb tangles and the consequences of the $R$ 's and $Q$ 's themselves. The main hard work of this paper is to calculate completely explicitly the orthogonal projection of $R \circ Q$ and all of its rotations onto the space spanned by these annular consequences when the subfactor planar algebra is $(n-1)$ supertransitive. We will see that the only parameters for this calculation are the structure constants for the algebra $P_{n}$ (in fact just the traces of cubic monomials) and the action of rotation.

By itself this projection is not very exciting and certainly does not justify the work but there are ways to exploit this knowledge. For instance we will see that for certain powers of the rotation $\rho$ it is possible to evaluate

$$
\left\langle R \circ Q, \rho^{k}(T \circ S)\right\rangle
$$

using planar manipulations. The inner products between the projections onto annular consequences are given by the annular theory which leads to non-trivial identities and inequalities.

The first version of this paper was available in preprint form as early as 2003 . It was one of the contributing factors to a project to classify all subfactors of index $\leq 5$ which involves several people including Bigelow, Morrison, Peters and Snyder (see [18],[2]). This project in turn influenced more recent versions of this paper and changed its emphasis somewhat. The author would like to gratefully acknowledge the input of all the people involved in this classification project. In particular theorem 5.2.2 was inspired by the classification project but we include it here because we wanted to show that the quadratic tangle technique applies more generally than in [16].

In section 5 we treat concrete examples, obtaining obstructions for graphs to be principal graphs of subfactors, and some new information about the Haagerup and Haagerup-Asaeda subfactors.

Throughout this paper we will use diagrams with a small number of strings to define tangles where the number of strings is arbritrary. This is a huge savings in notation and we shall strive to use enough strings so that the general situation is clear. 


\section{Planar Algebras}

The definition of a planar algebra has evolved a bit since the original one in [13] so we give a detailed definition which is, we hope, the ultimate one, at least for shaded planar algebras.

\subsection{Planar Tangles.}

Definition 2.1.1. Planar $k$-tangles.

$A$ planar $k$-tangle will consist of a smooth closed disc $D_{0}$ in $\mathbb{C}$ together with a finite (possibly empty) set $\mathcal{D}$ of disjoint smooth discs in the interior of $D$. Each disc $D \in \mathcal{D}$, and $D_{0}$, will have an even number $2 k_{D} \geq 0$ of marked points on its boundary with $k=k_{D_{0}}$. Inside $D_{0}$, but outside the interiors of the $D \in \mathcal{D}$, there is also a finite set of disjoint smoothly embedded curves called strings which are either closed curves or whose boundaries are marked points of $D_{0}$ and the $D \in \mathcal{D}$. Each marked point is the boundary point of some string, which meets the boundary of the corresponding disc transversally. The connected components of

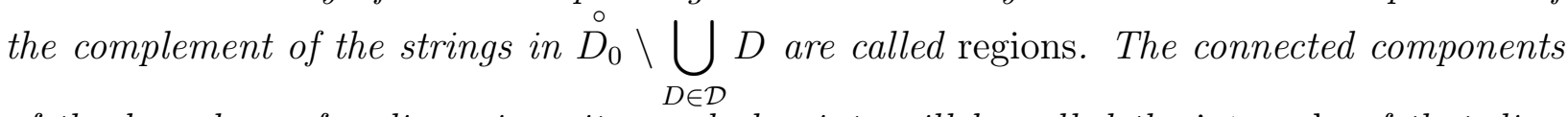
of the boundary of a disc minus its marked points will be called the intervals of that disc. The regions of the tangle will be shaded so that regions whose boundaries meet are shaded differently. The shading will be considered to extend to the intervals which are part of the boundary of a region.

Finally, to each disc in a tangle there is a distinguished interval on its boundary(which may be shaded or not).

Remark 2.1.2. Observe that smooth diffeomorphisms of $\mathbb{C}$ act on tangles in the obvious way-if $\phi$ is such a diffeomorphism, which may be orientation reversing, and $I$ is the distinguished boundary interval of a disc $D$ in the tangle $T$, then $\phi(I)$ is the distinguished boundary interval of $\phi(D)$ in $\phi(T)$.

Definition 2.1.3. The set of all planar $k$-tangles for $k>0$ will be called $\mathcal{T}_{k}$. If the distinguished interval of $D_{0}$ for $T \in \mathcal{T}$ is unshaded, $T$ will be called positive and if it is shaded, $T$ will be called negative. Thus $\mathcal{T}_{k}$ is the disjoint union of sets of positive and negative tangles: $\mathcal{T}_{k}=\mathcal{T}_{k,+} \sqcup \mathcal{T}_{k,-}$.

We will often have to draw pictures of tangles. To indicate the distinguished interval on the boundary of a disc we will place a ${ }^{*}$, near to that disc, in the region whose boundary contains the distinguished interval. To avoid confusion we will always draw the discs of a tangle as round circles and avoid round curves for closed strings. An example of a positive 4-tangle illustrating all the above ingredients is given below. 


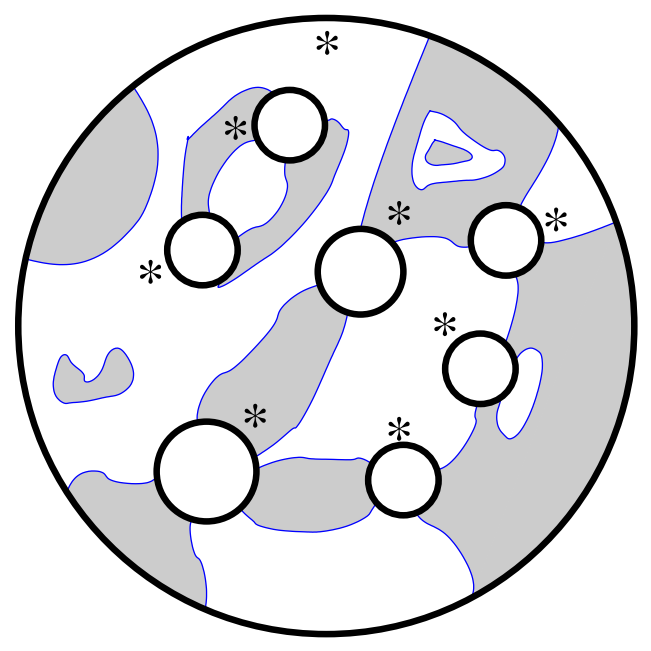

\subsection{Operadic Structure}

Planar tangles admit a partially defined "gluing" or "composition" operation which we now define.

Suppose we are given planar $k$ and $k^{\prime}$-tangles $T$ and $S$ respectively, and a disk $D$ of $T$ which is identical to the $D_{0}$ of $S$ as a smoothly embedded curve with points and shaded intervals. If we obtain a $k$-tangle as the union of the strings and discs of $S$ and $T$, excepting the disc $D$, we call that $k$-tangle $T \circ_{D} S$. Otherwise the gluing is not defined.

We exhibit the comoposition of tangles in the picture below.

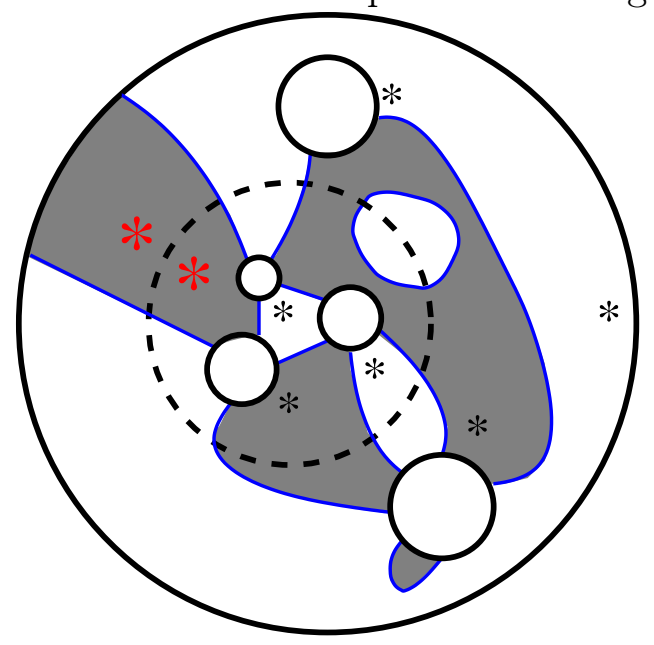

Here the tangle $S$ is the dotted circle and everything inside it, the tangle $T$ is the dotted circle and everything outside it and inside the outer circle which is its $D_{0}$. But the dotted circle is not part of a disc of $T \circ_{D} S$. The large (red) *'s are in $S$ and $T$ but not in $T \circ_{D} S$. 


\subsection{Planar Algebras}

Before giving the formal definition of a planar algebra we recall the notion of the cartesian product of vector spaces over an index set $\mathcal{I}, \quad \times_{i \in \mathcal{I}} V_{i}$. This is the set of functions $f$ from $\mathcal{I}$ to the union of the $V_{i}$ with $f(i) \in V_{i}$. Vector space operations are pointwise. Multilinearity is defined in the obvious way, and one convert multinearity into linearity in the usual way to obtain $\otimes_{i \in \mathcal{I}} V_{i}$, the tensor product indexed by $\mathcal{I}$.

Definition 2.3.1. Planar algebra.

$A$ (shaded) planar algebra $\mathcal{P}$ will be a family of $\mathbb{Z} / 2 \mathbb{Z}$-graded vector spaces indexed by the set $\{\mathbb{N} \cup\{0\}\}$, where $P_{k, \pm}$ will denote the \pm graded space indexed by $k$. To each planar $k$-tangle $T$ for $k \geq 0$ and $\mathcal{D}_{T}$ non empty, there will be a multilinear map

$$
Z_{T}: \times_{i \in \mathcal{D}_{T}} P_{i} \rightarrow P_{D_{0}}
$$

where $P_{D}$ is the vector space indexed by half the number of marked boundary points of $i$ and graded by + if the distinguished interval of $D$ is unshaded and - if it is shaded.

The map $Z_{T}$ is called the "partition function" of $T$ and is subject to the following two requirements:

(i) (Isotopy invariance) If $\varphi$ is an orientation preserving diffeomorphism of $\mathbb{C}$ then

$$
Z_{T}=Z_{\varphi(T)}
$$

where the sets of internal discs of $T$ and $\varphi(T)$ are identified using $\varphi$.

(ii)(Naturality)If $T \circ_{D} S$ exists and $\mathcal{D}_{S}$ is non-empty,

$$
Z_{T \circ_{D} S}=Z_{T} \circ_{D} Z_{S}
$$

Where $D$ is an internal disc in $T$, and to define the right hand side of the equation, first observe that $\mathcal{D}_{T_{{ }_{0}} S}$ is the same as $\left(\mathcal{D}_{T}-\{D\}\right) \cup \mathcal{D}_{S}$. Thus given a function $f$ on $\mathcal{D}_{T \circ_{D} S}$ to the appropriate vector spaces, we may define a function $\tilde{f}$ on $\mathcal{D}_{T}$ by

$$
\tilde{f}(E)= \begin{cases}f(E) & \text { if } E \neq D \\ Z_{S}\left(\left.f\right|_{\mathcal{D}_{S}}\right) & \text { if } E=D\end{cases}
$$

Finally the formula $Z_{T} \circ_{D} Z_{S}(f)=Z_{T}(\tilde{f})$ defines the right hand side.

The unital property could no doubt be included as part of the above definition by careful consideration of the empty set but we prefer to make it clear by doing it separately.

Definition 2.3.2. A shaded planar algebra will be called unital if for every planar $(k, \pm)$ tangle $S$ without internal discs there is an element $Z_{S} \in P_{k, \pm}$, depending on $S$ only up to isotopy, such that if $T \circ_{D} S$ exists then

$$
Z_{T \circ_{D} S}(f)=Z_{T}(\tilde{f})
$$


where

$$
\tilde{f}(E)= \begin{cases}f(E) & \text { if } E \neq D \\ Z_{S} & \text { if } E=D\end{cases}
$$

Remark 2.3.3. It is sometimes convenient to work with just one of $P_{n,+}$ and $P_{n,-}$. We agree on the convention that $P_{n}$ will mean $P_{n,+}$.

It follows from the axioms that in any unital planar algebra $\mathcal{P}$ the linear span of the $Z_{S}$ as $S$ runs through all planar tangles with no internal discs, forms a unital planar subalgebra.

Here is a basic example of a unital shaded planar algebra.

Example 2.3.4. The Temperley Lieb algebra TL.

Let $\delta$ be an arbitrary element of the field $K$. We will define a planar algebra $T L(\delta)$ or just $T L$ for short. We must first define the vector spaces $T L_{k, \pm}$. We let $T L_{k,+}$ be the vector space whose basis is the set of all isotopy classes of connected $k$-tangles with no internal discs, and for which the distinguished interval on the boundary is unshaded. (Here "connected" simply means as a subset of $\mathbb{C}$, i.e. there are no closed strings.) It is well known that the dimension of $T L_{k,+}$ is the Catalan number $\frac{1}{k+1}\left(\begin{array}{c}2 k \\ k\end{array}\right)$. Similary define $T L_{k,-}$, requiring the disitinguished interval to be shaded.

The definition of the maps $Z_{T}$ is transparent: a multilinear map is defined on basis elements so given a $k$-tangle $T$ it suffices to define a linear combination of tangles, given basis elements associated to each internal disc of $T$. But the basis elements are themselves tangles so they can be glued into their corresponding discs as in the composition of tangles once they have been isotoped into the correct position. The resulting tangle will in general not be connected as some closed strings will appear in the gluing. Just remove the closed strings, each time multiplying the tangle by $\delta$, until the tangle is connected. This multiple $\tilde{T}$ of a basis element is the result of applying $Z_{T}$ to the basis elements associated to the internal discs.

For the unital structure, if the tangle $S$ has no internal discs we put $Z_{S}=\tilde{S}$.

Remark 2.3.5. In the example above there is a constant $\delta \in K$ with the property that the partition function of a tangle containing a closed contractible string is $\delta$ times that of the same tangle with the string removed. We shall call such a planar algebra a planar algebra with parameter $\delta$.

\subsection{Labelled Tangles}

If $\mathcal{P}$ is a planar algebra and $T$ a planar tangle, and we are given elements $x_{D} \in P_{D}$ for $D$ 's in some subset $\mathcal{S}$ of the internal discs $\mathcal{D}_{T}$ of $T$ (with $P_{D}$ as in 2.3.1), we form the "labelled tangle" $T_{x_{D}}$ by writing each $x_{D}$ in its $D$ and then forming the linear map

$$
Z_{T_{x_{D}}}: \bigotimes_{D^{\prime} \in \mathcal{D}_{T} \backslash \mathcal{S}} P_{D^{\prime}} \rightarrow P_{D_{0}}
$$

in the obvious way.

Here is an example of a labelled tangle which defines a map from $P_{2,+} \otimes P_{3,-}$ to $P_{1,+}$. 
Fig. 2.4.1.

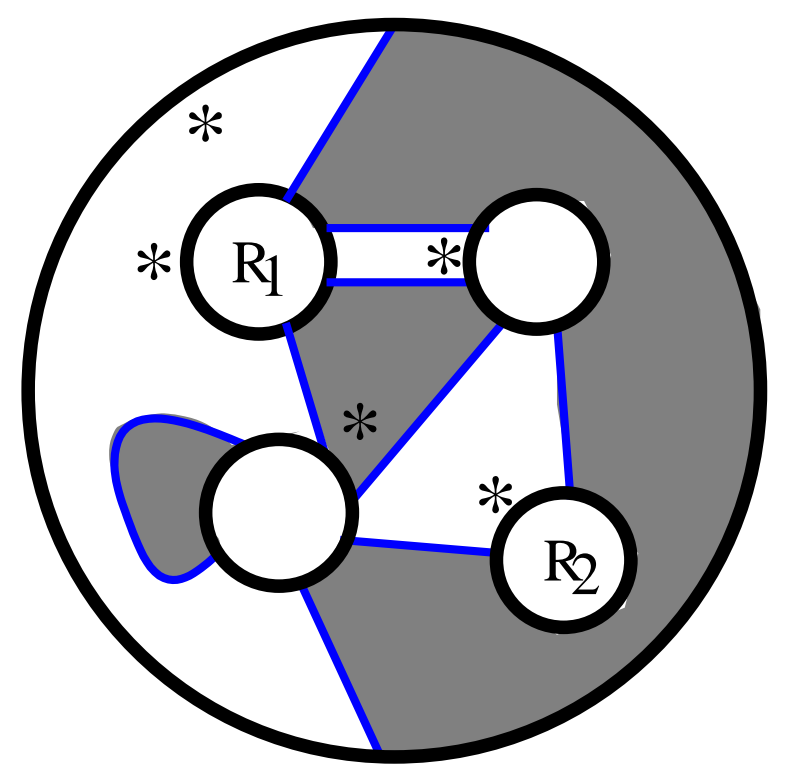

We will say that $T$ is fully labelled if $S=\mathcal{D}_{T}$ in the above.

The special case where $\operatorname{dim} P_{0, \pm}=1$ is common. In this case we will typically leave out the external boundary disc from the diagram-see for example 1.0.1. We will also leave out the shading when it is defined by other knowledge. For instance in 1.0.1, since we know that $a$ and $b$ are in $P_{3,+}$ the shading is defined by the distinguished intervals indicated with $*$ 's.

Spherical invariance is most easily expressed in terms of labelled tangles. We say that a planar algebra $\mathcal{P}$ is spherical if $\operatorname{dim} P_{0, \pm}=1$ and the partition function of any fully labelled 0 -tangle is invariant under spherical isotopy. Note that spherical isotopies can pass from tangles with the outside region shaded to ones where it is unshaded.

\subsection{Star Structure}

Another natural operation on planar tangles will be crucial in $C^{*}$-algebra considerations. We assume the field is $\mathbb{R}$ or $\mathbb{C}$.

Definition 2.5.1. We will say that a planar algebra $P$ is $a^{*}$-planar algebra if there each $P_{n, \pm}$ possesses a conjugate linear involution * and If $\theta$ is an orientation reversing diffeomorphism of $\mathbb{C}$, then $Z_{\theta(T)}\left(f \circ \theta^{-1}\right)=Z_{T}\left(f^{*}\right)^{*}$.

The Temperley-Lieb planar algebra is a planar *-algebra if $\delta \in \mathbb{R}$ and the involution on each $P_{n, \pm}$ is the conjugate-linear extension of complex conjugation acting on tangles.

\subsection{Subfactor planar algebras.}

Definition 2.6.1. A subfactor planar algebra $\mathcal{P}$ will be a spherical planar *algebra with

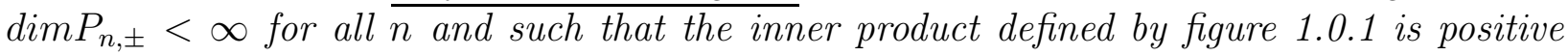
definite for all $n$ and grading \pm . 
It was shown in [13] that a subfactor planar algebra is the same thing as the standard invariant of a finite index extremal subfactor of a type $\mathrm{II}_{1}$ factor.

Here are some well known facts concerning subfactor planar algebras.

(i) The parameter $\delta$ is $>0$. The spaces $P_{0, \pm}$ are identified with $\mathbb{C}$ by identifying the empty tangle with $1 \in \mathbb{C}$.

(ii) Algebra structures on $P_{n, \pm}$ are given by the following tangle (with both choices of shading).

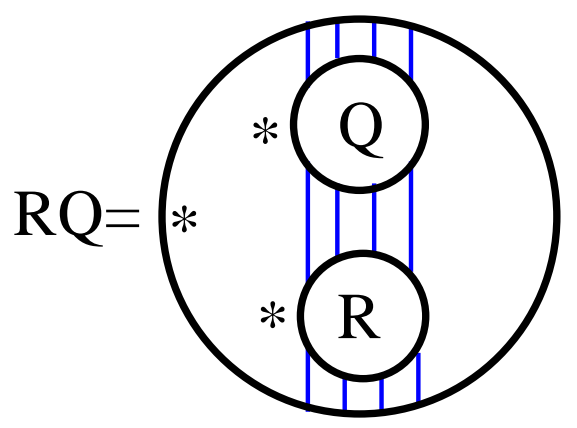

(iii) There is a pair of pointed bipartite graphs, called the principal graphs $\Gamma$, $*$ and $\Gamma^{\prime}$, * such that $P_{n,+}$ and $P_{n,-}$ have bases indexed by the loops of length $2 n$ based at $*$ on $\Gamma$ and $\Gamma^{\prime}$ respectively. The multiplication of these basis elements is easily defined using the first half of the first loop and the second half of the second, assuming the second half of the first is equal to the first half of the second (otherwise the answer is zero).

(iv) The tangle below (with both choices of shading) give vector space isomporphisms between $P_{n,+}$ and $P_{n,-}($ for $n>0)$ called the "Fourier transforms".

Fig. 2.6.2.

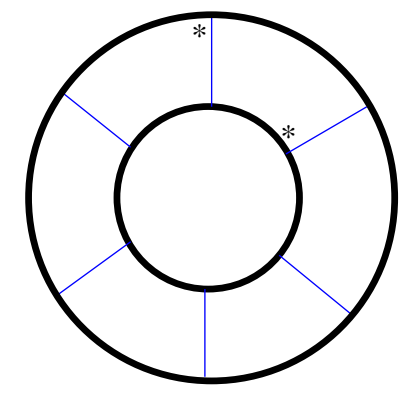

In particular one may pull back the multiplication on $P_{n,-}$ to $P_{n,+}$. If $n$ is odd the

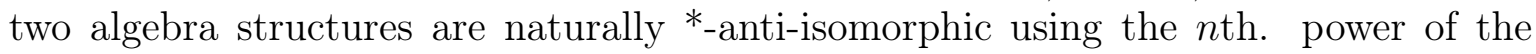
Fourier transform tangle $\mathcal{F}$. This allows us to identify the minimal central projections of $P_{n,+}$ with those of $P_{n,-}$. If $n$ is even the $n$ th. power of $\mathcal{F}$ gives anti-isomorphisms from $P_{n,+}$ and $P_{n,-}$ to themselves defining a possibly non-trivial bijection between the central projections. From a more representation-theoretic point of view these central projections correspond to bimodules and these maps are the "contragredient" maps. 
(v) The "square" of the Fourier transform acts on each $P_{n, \pm}$ and will be of vital importance for this paper. We will call it the rotation $\rho$ :

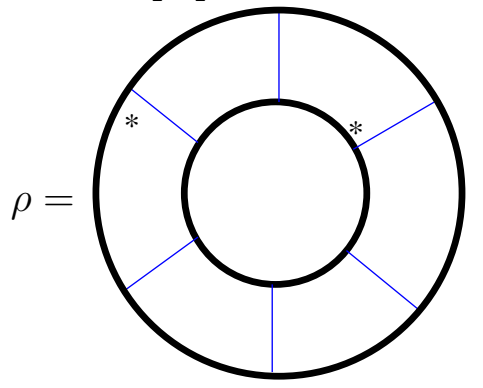

(vi) $\Gamma$ is finite iff $\Gamma^{\prime}$ is in which case $\delta$ is the norm of the adjacency matrix of $\Gamma$ and $\Gamma^{\prime}$ and the subfactor/planar algebra is said to have finite depth.

(vii) There is a trace $\operatorname{Tr}$ on each $P_{n, \pm}$ defined by the following 0 -tangle: $\operatorname{Tr}(R)=$

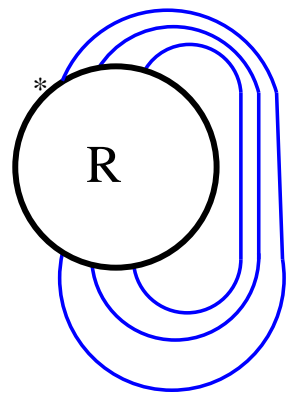

The innner product $\langle R, S\rangle$ on $P_{n}$ of figure 1.0.1 is given alternatively by $\operatorname{Tr}\left(R^{*} S\right)$. Our convention is that it is linear in the second variable and antilinear in the first, as implied by 1.0 .1 .

The algebra $P_{n, \pm}$ is semisimple over $\mathbb{C}$ and its simple components are matrix algebras indexed by the vertices of the principal graph at distance $n$ from * The trace $\operatorname{Tr}$ is thus given by assigning a "weight" by which the usual matrix trace must be multiplied in each simple component. This multiple is given by the Perron-Frobenius eigenvector (thought of as a function on the vertices) of the adjaceny matrix of the principal graph, normalised so that the value at $*$ is 1 . Because of the bipartite structure care may be needed in computing this eigenvector. To be sure, one defines $\Lambda$ to be the (possibly non-square) bipartite adjacency matrix and constructs the eigenvector for the adjacency matrix $\left(\begin{array}{cc}0 & \Lambda \\ \Lambda^{t} & 0\end{array}\right)$ as $\left(\begin{array}{c}\Lambda v \\ \delta v\end{array}\right)$ where $v$ is the Perron Frobenius eigenvector (of eigenvalue $\delta^{2}$ ) of $\Lambda^{t} \Lambda$.

(viii) There are "partial traces" or "conditional expectations" the simplest of which is $\mathcal{E}$, the map from $P_{n, \pm}$ to $P_{n-1, \pm}$ defined by the following tangle: 
Fig. 2.6.3. $\mathcal{E}=$

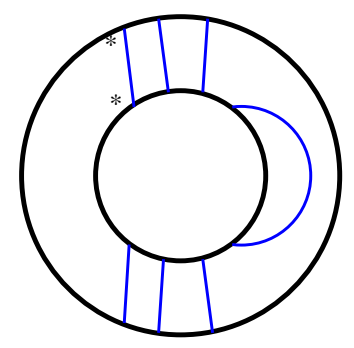

It is obvious that

$$
\operatorname{Tr}(\mathcal{E}(x))=\operatorname{Tr}(x) .
$$

(ix) The TL diagrams span a subalgebra of $P_{n,+}$ and $P_{n,-}$ which we will call TL for short. We will call $E_{i}$, for $1 \leq i \leq n-1$ the element of $P_{n, \pm}$ defined by the tangle below:

Fig. 2.6.4.

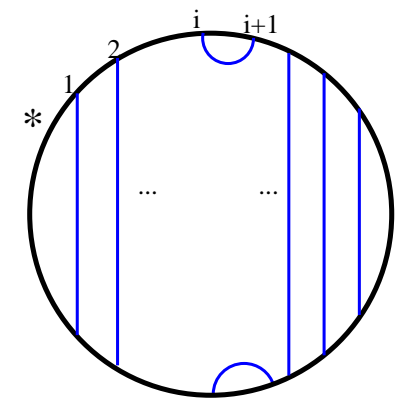

The algebra generated by the $E_{i}$ is a 2 -sided ideal in TL. Its identity is the JW projection $p_{n}$ which is uniquely defined up to a scalar and the property

\subsection{5.}

$$
\begin{array}{rlr}
E_{i} p_{n} & =p_{n} E_{i}=0 & \forall i<n \\
\operatorname{Tr}\left(x E_{n}\right) & =\operatorname{Tr}(x) & \text { for } x \in T L_{n}
\end{array}
$$

One has the formulae

\subsection{6.}

$$
\begin{aligned}
\operatorname{Tr}\left(p_{n}\right) & =[n+1] \\
\operatorname{Tr}\left(x E_{n}\right) & =\operatorname{Tr}(x) \text { for } x \in T L_{n} \\
\mathcal{E}\left(p_{n}\right) & =\frac{[n+1]}{[n]} p_{n-1} \\
p_{n+1} & =p_{n}-\frac{[n]}{[n+1]} p_{n} E_{n} p_{n}
\end{aligned}
$$


where $\delta=q+q^{-1}$ and $[r]$ is the quantum integer $\frac{q^{r}-q^{-r}}{q-q^{-1}}$. Note $[r+1]=\delta[r]-[r-1]$.

We will also use a formula from [14. Namely if $x$ is the $\mathrm{TL}_{n}$ element

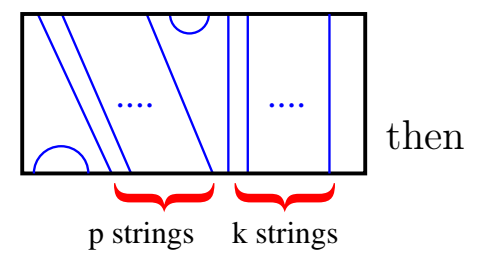

the coefficient of $x$ in $p_{n}$ is

2.6.7.

$$
(-1)^{p-1} \frac{[k+1]}{[n]}=(-1)^{p-1} \frac{[n-p-1]}{[n]}
$$

(x) Notation: the Fourier transform gives a canonical identification of $P_{n,+}$ with $P_{n,-}$ so one of the two is redundant data. It is convenient to set

$$
P_{n}=P_{n,+} \text {. }
$$

FOR THE REST OF THIS PAPER "PLANAR ALGEBRA" WILL MEAN "SUBFACTOR PLANAR ALGEBRA".

\subsection{Lowest weight vectors.}

In this paper a lowest weight vector $R$ will be an element of $P_{n}$ so that

(i) $\quad R^{*}=R$.

(ii)

Fig. 2.7.1.

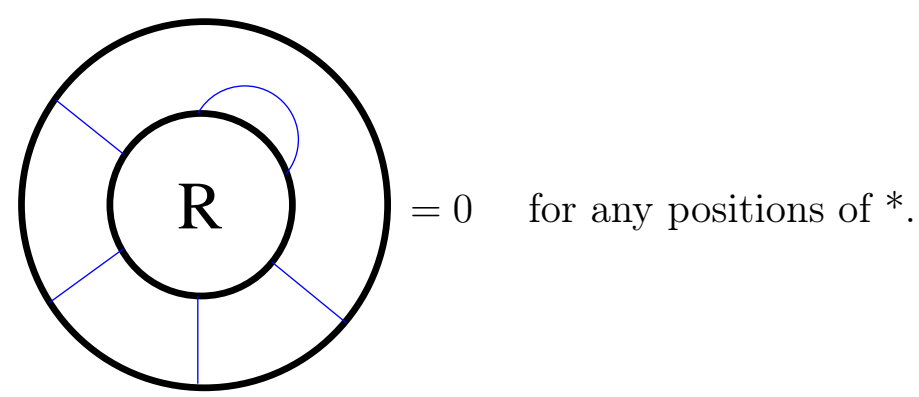

(iii)

Fig. 2.7.2. $\rho(R)=$

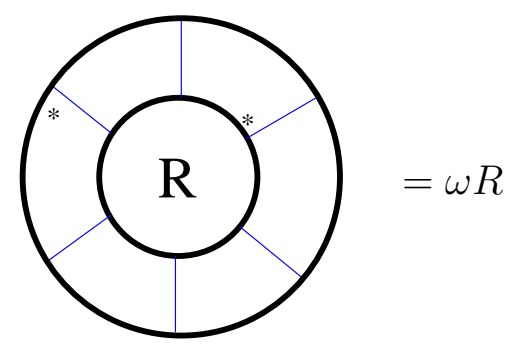

for some $n$ th. root of unity $\omega$. 
The diagram can be confusing to apply so let us say verbally what it means: "if you see a tangle with an input disc $D$ labelled $R$, then the tangle is $\omega$ times the same tangle but with the * of $D$ rotated counterclockwise by $2 "$.

As shown in [14], any planar algebra may be decomposed into an orthogonal direct sum of irreducible subspaces for the action of annular tangles, each irreducible summand being generated by a lowest weight vector.

\section{Supertransitivity and Annular multiplicity.}

Transitivity of a group action on a set $X$ is measured by the number of orbits on the Cartesian powers $X, X^{2}, X^{3}$, etc. The smallest number of orbits is attained for the full symmetric group $S_{X}$ and we say the group action is $k$-transitive if it has the same number of orbits on $X^{k}$ as does $S_{X}$. Transitivity can be further quantified by the number of orbits that an $S_{X}$ orbit breaks into on $X^{k}$ when the action fails to be $k$-transitive. There is a planar algebra $\mathcal{P}$ associated to a group action on $X$ for which $\operatorname{dim} P_{k}$ is the number of orbits on $X^{k}$. The planar algebra contains a copy of the planar algebra for $S_{X}$ and the action is $k$-transitive if $P_{k}$ is no bigger than the symmetric group planar algebra. Thus the symmetric group planar algebra is universal in this situation. We will call it the partition planar algebra. As a union of finite dimensional algebras it already appears in [15] and [17]. It depends on $X$ of course but only through $\#(X)$. Other planar algebras may be universal for other situations. The Fuss-Catalan subalgebra of [3] is such an example for subfactors which are not maximal. Another one, sometimes called the string algebra, is universal for the Wassermann subfactors for representations of compact groups. But the truly universal planar algebra in this regard is the TL planar algebra (a quotient of)which is contained in any planar algebra. Motivated by this discussion we will define a notion of supertransitivity measured by how small the algebra is compared to its TL subalgebra.

\subsection{The partition planar algebra.}

In [13] we defined a planar algebra $\mathcal{P}$, called the "spin model planar algebra" associated to a vector space $V$ of dimension $k$ with a fixed basis numbered $1,2, \ldots, k$. For $n>0 P_{n, \pm}$ is $\otimes^{n} V$ and $P_{0,+}=V, P_{0,-}=\mathbb{C}$. The planar operad acts by representing an element of $\otimes^{n} V$ as a tensor with $n$ indices (with respect to the given basis). The indices are associated with the shaded regions and summed over internal shaded regions in a tangle. There is also a subtle factor in the partition function coming from the curvature along the strings. This factor is only necessary to make a closed string count $\sqrt{k}$ independently of how it is shaded whereas without this factor a closed string would count $k$ if the region inside it is shaded and 1 otherwise. For more details see [13]. Nothing in the planar algebra structure differentiates between the basis vectors so the symmetric group $S_{k}$ acts on $P$ by planar

*-algebra automorphisms. 
Definition 3.1.1. If $G$ is a group acting on the set $\{1,2, \ldots, k\}$ we define $P^{G}$ to be the fixed point sub-planar algebra of the spin model planar algebra under the action of $G$. The partition planar algebra $\mathcal{C}=\left\{C_{n, \pm} \mid n=0,1,2, \ldots\right\}$ is $P^{S_{k}}$.

Remark 3.1.2. If $G$ acts transitively, passing to the fixed point algebra makes $\operatorname{dim} C_{0,+}=$ $\operatorname{dim} C_{0,-}=1$ and spherical invariance of the partition function is clear, as is positive definiteness of the inner product. So $P^{G}$ is a subfactor planar algebra. The subfactor it comes from is the "group-subgroup" subfactor- choose an outer action of $G$ on a $\mathrm{II}_{1}$ factor $M$ and consider the

subfactor $M^{G} \subseteq M^{H}$ where $H$ is the stabilizer of a point in $\{1,2, \ldots, k\}$.

Proposition 3.1.3. The action of $G$ is $r$-transitive iff $P_{r}^{G}=C_{r}$.

Proof. By definition the dimension of $P_{r, \pm}^{G}$ is the number of orbits for the action of $G$ on $\{1,2, \ldots, k\}^{r}$.

\subsection{Supertransitivity}

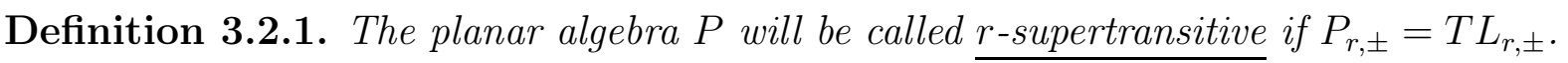

Example 3.2.2. The $D_{2 n}$ planar algebra with $\delta=2 \cos \pi /(2 n-2)$ is $r$-supertransitive for $r<2 n-2$ but not for $r=2 n-2$.

The following question indicates just how little we know about subfactors. Analogy with the Mathieu groups suggests that the answer could be interesting indeed.

Question 3.2.3. For each $r>0$ is there a (subfactor) planar algebra which is $r$-supertransitive but not equal to $T L($ for $\delta>2)$ ?

The question is even open for $r=4$ if we require that the planar algebra is not $(r+1)$ supertransitive.

Example 3.2.4. The partition planar algebra $\mathcal{C}$ is 3-supertransitive but not 4-supertransitve.

This is because there are just 5 orbits of $S_{X}$ on $X^{3}$ but 15 on $X^{4}$ (at least for $\#(X)>3$ ).

The record for supertransitivity at the time of writing is seven and is held by the "extended Haagerup" subfactor constructed in [2]. The Asaeda-Haagerup subfactor is 5-supertransitive.

\subsection{Annular multiplicity}

A subfactor planar algebra is always a direct sum of orthogonal irreducible modules for the action of the annular category-see [14]. The irreducible representations of the annular category are completely determined by their "lowest weight" and rotational eigenvalue. For a planar algebra, in practical terms this means that each $P_{n}$ contains a canonical subspace $A C_{n}$ which is the image of the annular category on $P_{n-1}$. Elements of $A C_{n}$ are called "annular consequences" of elements in $P_{k}, k<n$. 
Definition 3.3.1. If $\mathcal{P}$ is a planar algebra the number

$$
a_{n}=\operatorname{dim} P_{n}-\operatorname{dim} A C_{n}
$$

for $n>0$ is called the $n$th annular multiplicity.

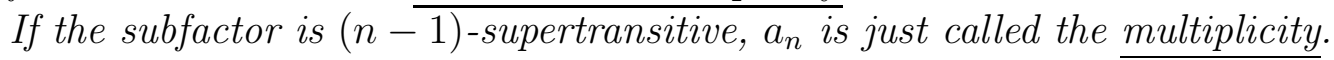

Obviously $k$-supertransitivity is equivalent to $a_{r}=0$ for $r \leq k$ but it is quite possible for $a_{r}$ to be zero without $r$-supertransitivity. There is an explicit formula for the generating function of the $a_{n}$ in terms of the generating function for the dimensions of the planar algebra itself in [14]. We will record the annular multiplicity as a sequence. For instance the multiplicity sequence for the partition planar algebra begins $00010 \ldots$. Obviously all the 0's up to the supertransitivity have no interest and we will be interested in situations where that sequence of zeros can have arbitrary length so we will abbreviate the sequence by compressing the leading zeros to $\mathrm{a}^{*}$. Thus the annular multiplicity sequence for the partition planar algebra would begin $* 10$. Indeed our real applications to subfactors will concern those with multiplicity sequence beginning $* 10$ or $* 20$. If we wish to give the leading zeros as well we will use the notation $0^{k} a_{k+1} a_{k+2}$ for a sequence beginning with $k$ zeros followed by $a_{k+1} a_{k+2}$ followed by anything at all.

We will allow this terminology to refer to either a subfactor or its planar algebra.

\subsection{Chirality.}

If a planar algebra is $n-1$-supertransitive with $n$ th. annulary multiplicity equal to $k$, the rotation acts on the othogonal complement of TL in the $n$-box space. This implies that planar algebras may have a "handedness". Over the complex numbers one may diagonalise the rotation on this orthogonal complement to obtain a family of $n$-th. roots of unity. These numbers will be called the "chirality" of the planar algebra.

\subsection{Examples}

Example 3.5.1. The $D_{2 n}$ planar algebra with $\delta=2 \cos \pi /(2 n-2)$ has annular multiplicity sequence beginning $0^{n} 10$. It has multiplicity one. The principal graphs are both (graphs with $2 n$ vertices)

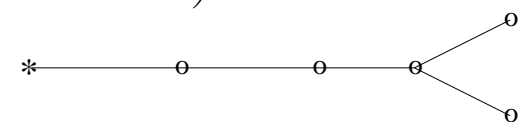

The chirality of this example was calculated in [13]. It is -1 .

Example 3.5.2. The Haagerup subfactors of index $\frac{5+\sqrt{13}}{2}$ have multiplicity sequence $0^{3} 10$. They have multiplicity one and are 3-supertransitive. 
The principal graphs are

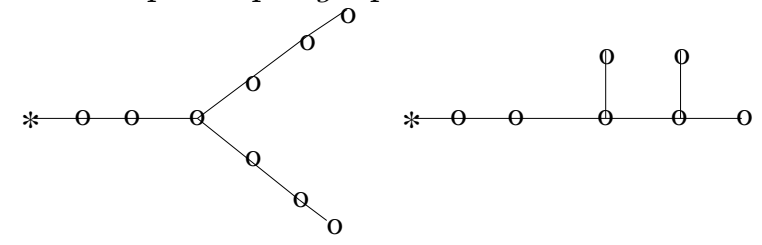

Once a principal graph is known it is easy enough to calculate all the annular multiplicities. For Haagerup the sequence begins $0^{3} 1010$. We will see that the chirality is -1 .

Example 3.5.3. The partition planar algebra has multiplicity sequence $0^{3} 10$.

This follows from the statement that the principal graphs for the partition planar algebra and the Haagerup one are the same for distance $\leq 5$ from *

Let us calculate both principal graphs for $\mathcal{C}$ (for $k>4$ ) to distance 5 from ${ }^{*}$, together with the weights of the trace $T r$ which we will use later. Our method will be a bit ad hoc but adapted to the needs of this paper. By counting orbits we know there are two central projections $e$ and $f$ orthogonal to the basic construction in $\mathcal{C}_{4}$. This is the same as saying that the principal graphs are both as below at distance $\leq 4$ from *:

Fig. 3.5.4.

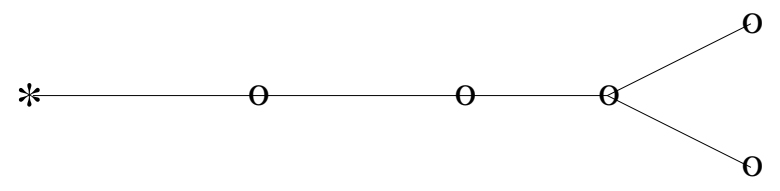

Our first step will be to calculate $\alpha=\operatorname{Tr}(e)$ and $\beta=\operatorname{Tr}(f)$. We know that

$$
\mathcal{C}_{4,+} \cong M_{2}(\mathbb{C}) \oplus M_{3}(\mathbb{C}) \oplus e \mathbb{C} \oplus f \mathbb{C}
$$

and that the trace of a minimal projection in $M_{2}(\mathbb{C})$ is 1 , that of a minimal projection in $M_{3}(\mathbb{C})$ is $\delta^{2}-1$ and that

3.5.5.

$$
\alpha+\beta=\delta^{4}-3 \delta^{2}+1
$$

To obtain another relation on $\alpha$ and $\beta$ one may proceed as follows. By [15] $\mathcal{C}_{4,+}$ is spanned by TL and the flip transposition $S$ on $V \otimes V$. With attention to normalisation one gets $\operatorname{Tr}(S)=\delta^{2}(=k)$. But in the 2-dimensional representation $S$ is the identity and in the 3 -dimensional one it fixes one basis element and exchanges the other two so it has trace 1. We may assume $e S=e$ and $f S=-f$ for if both reductions had the same sign $S$ would be in TL. Thus

3.5.6.

$$
\alpha-\beta+2+\left(\delta^{2}-1\right)=\delta^{2}
$$

Combining this with 3.5 .5 we obtain
3.5.7.

$$
\alpha=\frac{\delta^{4}-3 \delta^{2}}{2} \quad \beta=\frac{\delta^{4}-3 \delta^{2}+2}{2}
$$


We may now repeat the argument for $\mathcal{C}_{4,-}$. It is easy to check that $\mathcal{F}(S)$ is a multiple of a projection which is in the centre. It is the identity in the 2-dimensional representation and zero in the 3-dimensional one. The trace of the projection is $\delta^{2}$ and it must be zero on one of the projections orthogonal to TL and 1 on the other. Thus the equation corresponding to 3.5.6 becomes simply

$$
2+\alpha=\delta^{2} .
$$

Combining this with 3.5.5 we obtain

$$
\text { 3.5.8. } \alpha=\delta^{2}-2 \quad \beta=\delta^{4}-4 \delta^{2}+3
$$

Now we can deduce the algbebra structures of $\mathcal{C}_{4, \pm}$. By counting orbits we see that the principal graphs to distance five must have precisely two extra vertices (for $k>4$ ). But since the traces form an eigenvector for the adjacency matrix, nothing else can be attached to the vertex with trace $\delta^{4}-2$ in the graph for $\mathcal{C}_{4,-}$, and something must be attached to both vertices for $\mathcal{C}_{4,+}$. The conclusion is that the two principal graphs are as below to distance 5 from *.
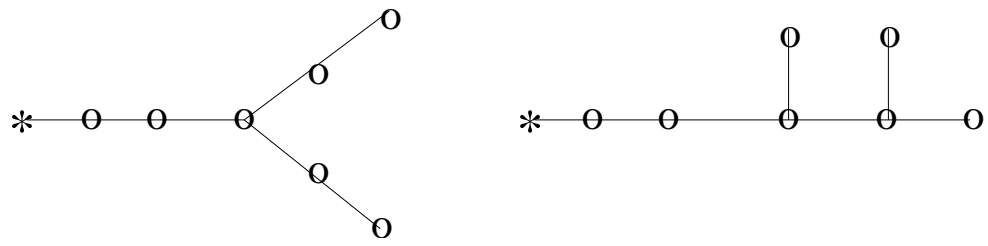

By counting orbits one may calculate the chirality of the partition planar algebra. It is -1 .

Note the identity between the principal graphs of the Haagerup subfactor and the partition planar algebra for distance up to five from *. The partition algebra has been studied by several people $([17,, 9])$ with generic values of the parameter $k$. One might be tempted to think that the Haagerup subfactor is some kind of specialisation of the partition algebra but this is not at all the case. For the chiralities are -1 (Haagerup) and +1 (partition), so these two planar algberas must be considered very distant cousins indeed.

Example 3.5.9. Haagerup-Asaeda. The principal graphs and traces are in [1]. The annular multiplicity sequence begins $0^{5} 1010$. We will see that the chirality is +1 .

Example 3.5.10. Fuss-Catalan The planar algebra of [3] is 1-supertransitive. For generic values of the parameters $a$ and $b$ (e.g. $a>2, b>2$ the principal graph begins:

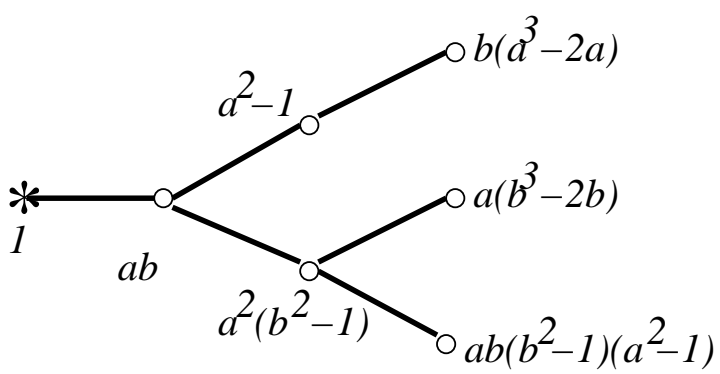


We have recorded the (non-normalised) traces of the minimal projections corresponding to the vertices of the graph. The dual principal graph is the same except that the roles of $a$ and $b$ are reversed.

The multiplicity sequence begins $* 11$. Observe however that for the special allowed value $a=\sqrt{2}$ the principal graph and dual principal graph begin:
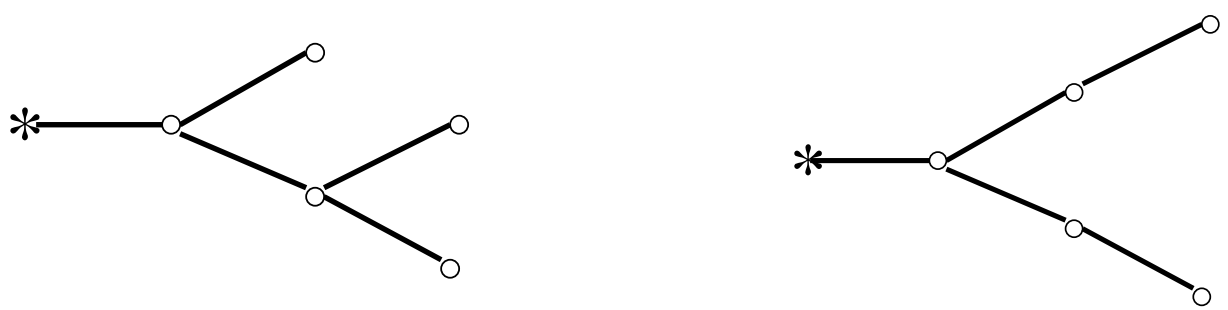

and the multiplicity sequence then begins $* 10$.

The biprojection generating the Fuss-Catalan algebra is rotationally invariant so the chirality of the algebra is +1 .

Remark 3.5.11. Observe that in all the examples whose annular multiplicity sequence begins $0^{k} 10$ the principal and dual principal graphs are different and begin like the Haagerup ones, but with a longer or shorter initial segment from $*$. We will explain this phenomenon with the quadratic tangle results.

\section{Inner product formulae.}

\subsection{Setup for this section.}

Let $\mathcal{P}$ have annular multiplicity beginning $0^{n-1} e$. The cyclic group of order $n$ acts unitarily on the $e$-dimensional orthogonal complement of the Temperley-Lieb subspace of $P_{n,+}$ via the rotation tangle. Choose an orthonormal basis $\mathfrak{B}=\{R\}$ of self-adjoint eigenvectors for the rotation with $\omega_{R}$ being the eigenvalue of $R \in \mathfrak{B}$. For each $R \in \mathfrak{B}, \mathcal{F}(R)^{*}=\omega^{-1} \mathcal{F}(R)$ so we choose a square root $\sigma_{R}$ of $\omega$ and define $\check{R}=\sigma^{-1} \mathcal{F}(R) \in P_{n,-}$ so that $(\check{R})^{*}=\check{R}$. Thus

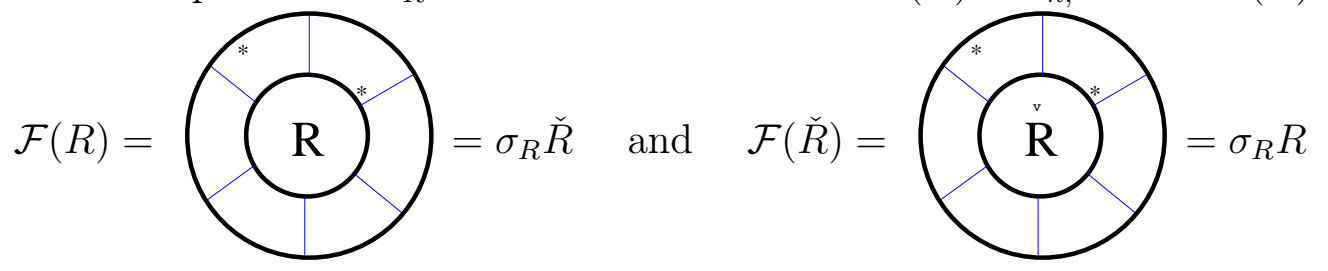

and we record the verbal version:

"If a tangle contains a disc $D$ labelled with $R$ (resp. $\check{R}$ ) then it is the same as $\sigma_{R}$ times the same tangle with the * of $D$ rotated counterclockwise by one, and $R$ (resp. $\check{R}$ ) in $D$ replaced by $\check{R}$ (resp. R)."

Remark 4.1.1. As we have defined them in 4.1, all the $R$ 's $\check{R}$ 's and $\sigma$ 's could be changed by a sign. One can do better than this under appropriate circumstances. For instance if 
the traces of both $R^{3}$ and $(\check{R})^{3}$ (which are necessarily real) are non-zero, one can impose the choice of $R$ and $\check{R}$ which makes both these traces positive. Then $\sigma_{R}$ is an unambiguous 2 nth. root of unity. But this does not always happen, even in the Haagerup subfactor, so we must live with the sign ambiguity. Of course any constraints we obtain must depend only on $\omega_{R}$.

\subsection{Inner products of annular consequences}

Definition 4.2.1. Let $\mathfrak{A}$ be the subspace of $P_{n+1,+}$ spanned by the image of $\mathfrak{B}$ under the annular Temperley-Lieb tangles.

We will use two bases for $\mathfrak{A}$ - carefully chosen annular consequences of the $R \in \mathfrak{B}$ and the dual basis. (That the annular images are linearly independent is shown in [14.)

Definition 4.2.2. In $P_{n+1,+} \oplus P_{n+1,-}$ let
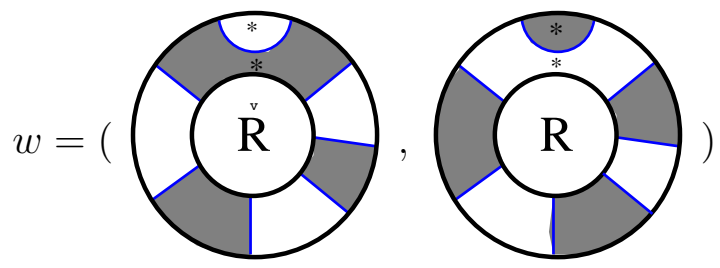

Let $\pi_{ \pm}$be the projections onto the first and second components of $P_{n+1,+} \oplus P_{n+1,-}$ and for $i \in \mathbb{Z} /(2 n+2) \mathbb{Z}$ define the following elements of $P_{n+1,+}$ :

$$
\cup_{i} R=\pi_{+}\left(\mathcal{F}^{i}(w)\right)
$$

Proposition 4.2.3. We have the following inner product formulae:

(a)

$$
\begin{aligned}
\text { (a) } & \left\langle\cup_{i} R, \cup_{i} R\right\rangle & =\delta \\
\text { (b) } & \left\langle\cup_{i} R, \cup_{i \pm 1} R\right\rangle & =\sigma_{R}^{ \pm 1}
\end{aligned}
$$

All other inner products among the $\cup_{i} R$ are zero .

Proof. Since $\mathcal{F}$ is unitary and $\mathcal{F} \pi_{+}=\pi_{-} \mathcal{F}$,

$$
\left\langle\pi_{+} \mathcal{F}^{i}(w), \pi_{+} \mathcal{F}^{j}(w)\right\rangle=\left\langle\pi_{ \pm}(w), \pi_{ \pm} \mathcal{F}^{j-i}(w)\right\rangle
$$

the sign of $\pi$ depending on the parity of $i$. This is clearly zero if $j-i \notin\{-1,0,1\}$ and equal to $\delta$ if $i=j$. Both $\pi_{+}(w)$ and $\pi_{-}(w)$ are self-adjoint and the pictures for computing the remaining inner products are the same for both parities of $i$, up to changing shading and interchanging $R$ and $\check{R}$. We illustrate below with $\left\langle\cup_{0} R, \cup_{1} R\right\rangle$. (The shadings are implied by the ${ }^{*}$ 's and $R, \check{R}$ ): 


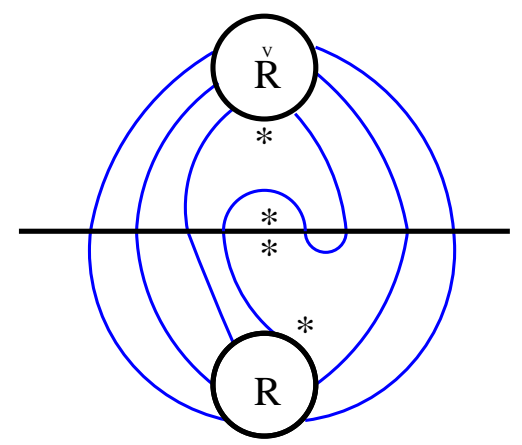

Corollary 4.2.4. The map $\rho^{1 / 2}: \mathfrak{A} \rightarrow \mathfrak{A}$ defined by

$$
\rho^{1 / 2}\left(\cup_{i} R\right)=\cup_{i+1} R
$$

(for $i \in \mathbb{Z} /(2 n+2) \mathbb{Z})$ is unitary. (And of course $\left(\rho^{1 / 2}\right)^{2}=\rho$.)

To project onto $\mathfrak{A}$ we will use the dual basis to $\mathfrak{B}$. Fortunately there is an elegant pictorial formula for the dual element $\hat{R}$ to $R$. First we define an unnormalised form of it.

Definition 4.2.5. For $R \in \mathfrak{B}$ as above let
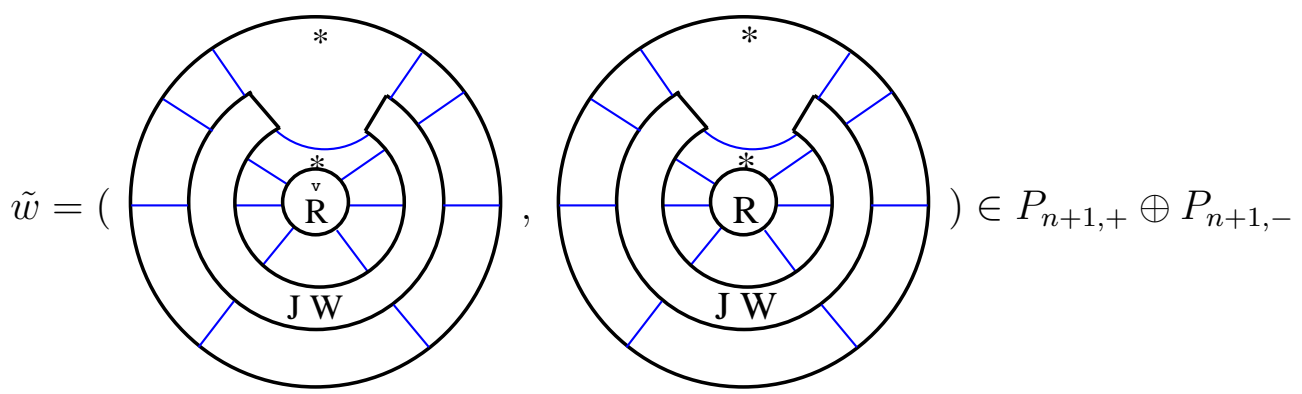

where in the crescent shaped areas we have inserted the appropriately shaded Jones-Wenzl idempotent $p_{2 n+2}$ (the shading is implicit from the position of star).

Definition 4.2.6. For an integer $k$ and $a \omega \neq 0$ define

$$
W_{k, \omega}(q)=q^{k}+q^{-k}-\omega-\omega^{-1} .
$$

For $R, \check{R}$ as above and for $i \in \mathbb{Z} /(2 n+2) \mathbb{Z}$ set

$$
\hat{\cup_{i}} R=\frac{[2 n+2]}{W_{2 n+2, \omega_{R}}(q)} \pi_{+}\left(\mathcal{F}^{i}(\tilde{w})\right)
$$

Lemma 4.2.7. For all $i$ and $j$,

$$
\left\langle\cup_{i} R, \cup_{j} R\right\rangle= \begin{cases}1 & i=j \\ 0 & i \neq j\end{cases}
$$


Proof. It is clear from the properties of the $p_{k}$ 's that the only non-zero inner products occur as written. So it is only a matter of the normalisation, which by unitarity of the rotation reduces to showing $\left\langle\tilde{\cup_{0}} R, \cup_{0} R\right\rangle=\frac{W_{2 n+2, \omega_{R}}(q)}{[2 n+2]}$.

We draw this inner product below:

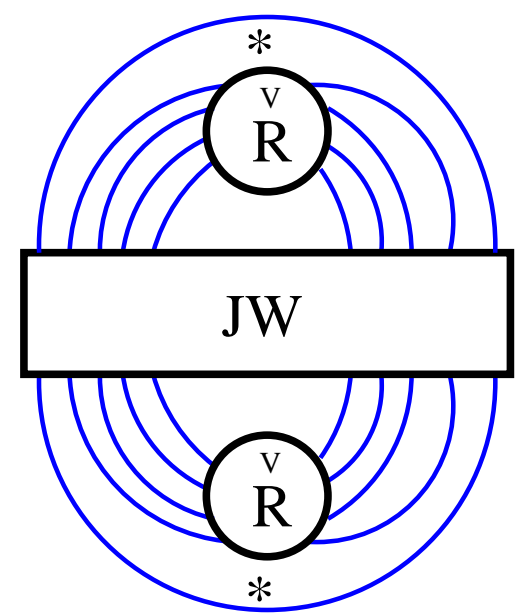

There are 5 TL elements that give non-zero contributions to the inner product. The first is the identity which clearly contributes $\delta$. The next four come in reflected pairs. The first pair contains one gets

$\frac{[2 n+2] \delta-2[2 n+1]-\omega-\omega^{-1}}{[2 n+2]}$ which is correct.

We now expand the JW idempotent to obtain an expression for the dual basis in terms of the $\cup_{i}(R)$. 
Proposition 4.2.8. With $W=W_{2 n+2, \omega_{R}}$ and $\sigma=\sigma_{R}$ we have $\hat{\cup_{0} R}=\frac{1}{W}\left\{[2 n+2] \cup_{0} R+\sum_{i=-n}^{+n}(-\sigma)^{n+i-1}([n+i+1]+\omega[n-i+1]) \cup_{n-i+1} R\right\}$

Proof. Consider the diagram for $\pi_{+}(\tilde{w})$ :

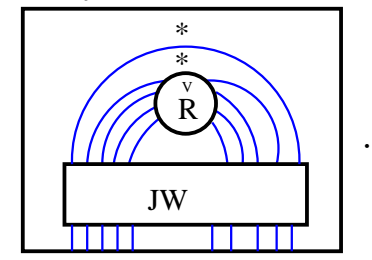

Caps at the top of the JW can only occur at the extreme left and

right but not at both. The only way to get a multiple of $\cup_{0} R$ is to take the identity in JW. The rest of the terms come in pairs, with caps at the top at the left and right, each with a cap at the bottom which we will index by $i$, the distance from the middle interval at the bottom. We illustrate below the two terms contributing to $\cup_{i} R$ with $i>0, i=2$ in the example (if $i$ were odd the only difference is that the internal box would contain $R$ rather than $\check{R}$ ) :
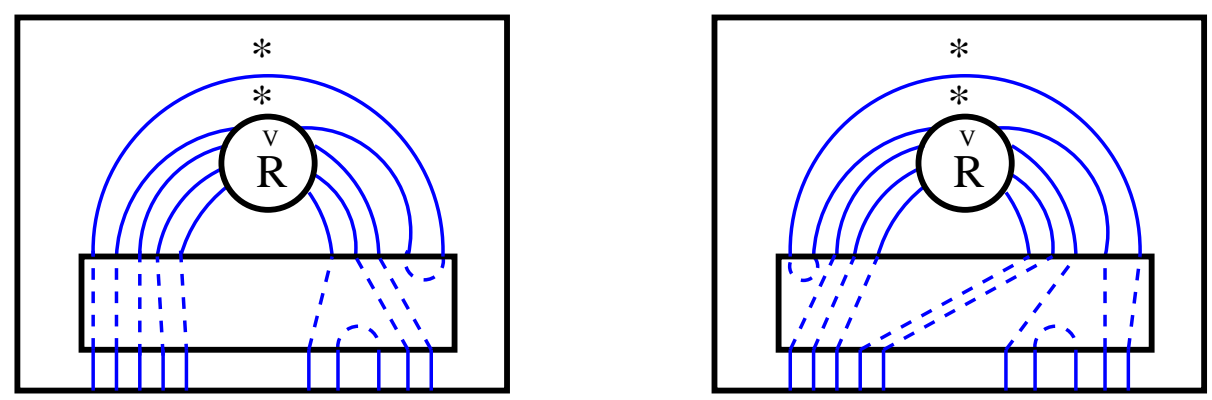

In both cases the starred boundary interval lies $n-i+1$ intervals counterclockwise from the cap on the outer boundary so both terms are multiples of $\cup_{n-i+1} R$. In the diagram on the left, the starred interval on the boundary of the disc containing $\check{R}$ needs to be rotated $(n+i-1)$ intervals counterclockwise to line up with $\cup_{n-i+1} R$ and the term inside the JW has " $p$ " $=n-i$ in the notation of 2.6 .7 whose coefficient is thus $\frac{[n+i+1]}{[2 n+2]}$. In the diagram on the left, the ${ }^{*}$ of $\check{R}$ must be rotated $n+i+1$ counterclockwise and the " $p$ " factor is $n+i$.

This establishes the terms in the sum for $i \geq 0$. Negative values of $i$ can be obtained by taking the adjoint and using $\left(\cup_{k} R\right)^{*}=\cup_{-k} R$.

All the other $\hat{\cup}_{i} R$ can be obtained from the above by applying a suitable power of $\rho^{1 / 2}$ but care needs to be taken with the indices as $\sigma_{R}$ is a $(2 n)$ th. root of unity and not a $(2 n+2)$ th.

The formula is so important that we record a few other versions of it. 
Proposition 4.2.9. (i)

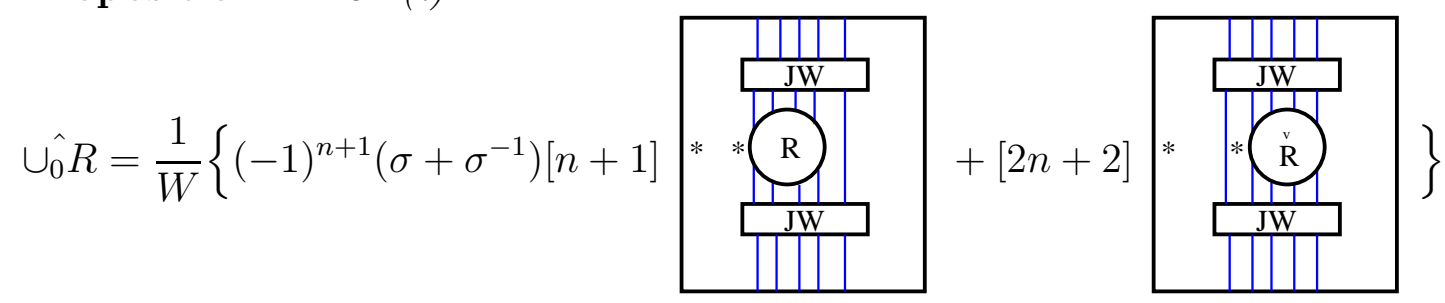

(ii) If $X=\sum_{j=1}^{n}(-\sigma)^{-j}(\omega[j]+[2 n+2-j]) \cup_{j} R$ then

$$
\hat{\cup_{0}} R=\frac{1}{W}\left\{[2 n+2] \cup_{0} R+\left((-\sigma)^{n+1}+(-\sigma)^{-n-1}\right)[n+1] \cup_{n+1} R+X+X^{*}\right\}
$$

(iii) If $Y=\sum_{j=1}^{n}(-\sigma)^{n-j-1}(\omega[n+j+1]+[n-j+1]) \cup_{j} R$ then

$$
\cup_{n+1} R=\frac{1}{W}\left\{[2 n+2] \cup_{n+1} R+\left((-\sigma)^{n+1}+(-\sigma)^{-n-1}\right)[n+1] \cup_{0} R+Y+Y^{*}\right\}
$$

For instance:

Corollary 4.2.10. $\cup_{n+1} R=\frac{[2 n+2]}{W_{2 n+2, \omega}} \cup_{n+1} R$

$$
+\frac{[2 n+2]}{W_{2 n+2, \omega_{R}}} \sum_{i=-n}^{+n}\left\{(-\sigma)^{n+i-1}[n+i+1]+(-\sigma)^{n+i+1}[n-i+1]\right\} \cup_{-i} R
$$

\subsection{Inner products between linear and quadratic tangles.}

We now define the main ingredients of this paper, certain quadratic tangles giving elements in $P_{n+1}$.

Definition 4.3.1. For $S, T \in \mathfrak{B}$ let

(i) $S \circ T=$

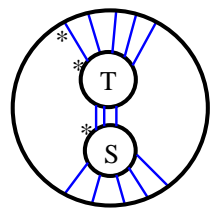

(ii) $S \star T=\mathcal{F}(\mathcal{F}(S) \circ \mathcal{F}(T))=\sigma_{S} \sigma_{T} \mathcal{F}(\check{S} \circ \check{T})=$

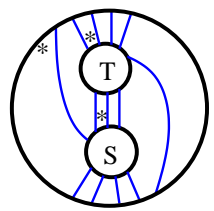

\subsection{Projection onto $\mathfrak{A}$.}

We can calculate $P_{\mathfrak{A}}$, the orthogonal projection onto $\mathfrak{A}$, for many quadratic tangles. 


\section{Proposition 4.4.1.}

$$
\begin{gathered}
\text { (i) } P_{\mathfrak{A}}(S \circ T)=\sum_{R \in \mathfrak{B}} \sigma_{R}^{n} \operatorname{Tr}(R S T) \hat{\cup_{n+1} R+\sigma_{T}^{-1} \sigma_{S} \operatorname{Tr}(\check{R} \check{S} \check{T}) \hat{\cup_{0} R}} \\
\text { (ii) } P_{\mathfrak{A}}(S \star T)=\sigma_{S} \sigma_{T} \sum_{R \in \mathfrak{B}} \sigma_{R}^{n} \operatorname{Tr}(\check{R} \check{S} \check{T}) \hat{\cup_{n+2}} R+\sigma_{T}^{-1} \sigma_{S} \operatorname{Tr}(R S T) \hat{\cup_{1}} R
\end{gathered}
$$

Proof. (i) Write $P_{\mathfrak{A}}(S \circ T)$ as a linear combination of the $\hat{\cup}_{i} R$. The coefficient of $\hat{\cup}_{i} R$ is just $\overline{\left\langle S \circ T, \cup_{i} R\right\rangle}$ which can only be non-zero if $i=0$ or $i=n+1$. These two cases are given by the following diagrams (for $n=4$ ):
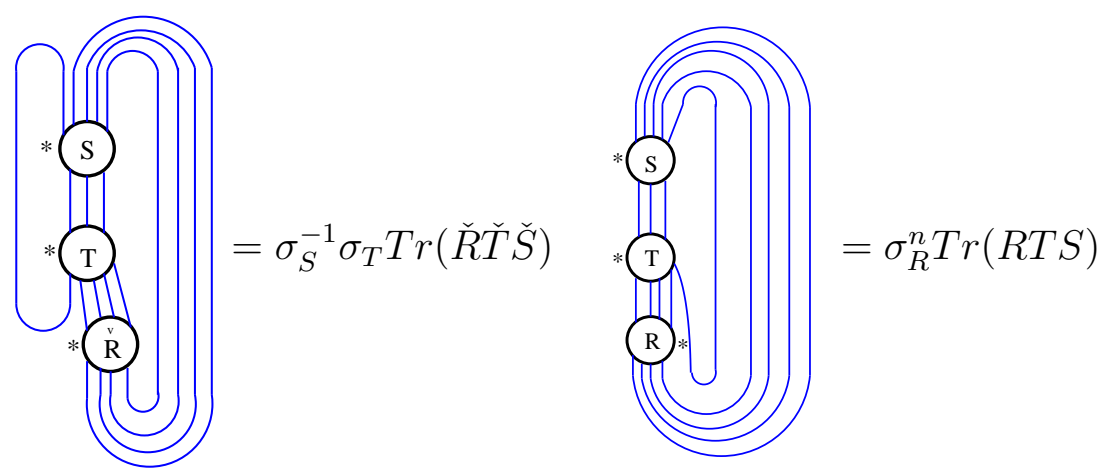

(ii) follows from (i) applied to the dual planar algebra, $S \star T=\sigma_{S} \sigma_{T} \mathcal{F}(\check{S} \circ \check{T})$, the fact that $\mathcal{F}$ interwines projection onto $\mathfrak{A}$ and the fact that $\sigma_{\hat{R}}=\sigma_{R}$ if we choose the basis $\check{R}$ in the dual.

We can now give a kind of "master formula" for inner products after projection onto $\mathfrak{A}$.

Proposition 4.4.2. Fix $P, Q, S, T \in \mathfrak{B}$ and for each $R \in \mathfrak{B}$ let

$$
a_{R}^{S T}=\operatorname{Tr}(R S T), b_{R}^{S T}=\operatorname{Tr}(\check{R} \check{S} \check{T})
$$

Then putting $W_{2 n+2, \omega_{R}}=W_{R}$, for $0 \leq 2 j \leq n$,

$$
\begin{aligned}
& \left\langle P_{\mathfrak{A}}(S \circ T), \rho^{j}(P \circ Q)\right\rangle= \\
& \sum_{R} \frac{\omega_{R}^{j}}{W_{R}}\left\{\left(\overline{a_{R}^{S T}} a_{R}^{P Q}+\sigma_{T} \bar{\sigma}_{S} \bar{\sigma}_{Q} \sigma_{P} \overline{b_{R}^{S T}} b_{R}^{P Q}\right)\left(\omega_{R}^{-1}[2 j]+[2(n-j)+2]\right)\right. \\
& \left.\quad+(-1)^{n+1} \sigma_{R}\left(\bar{\sigma}_{Q} \sigma_{P} \overline{a_{R}^{S T}} b_{R}^{P Q}+\sigma_{T} \bar{\sigma}_{S} \overline{b_{R}^{S T}} a_{R}^{P Q}\right)\left(\omega_{R}^{-1}[n+2 j+1]+[n-2 j+1]\right)\right\}
\end{aligned}
$$




$$
\begin{aligned}
& \text { and for } 0 \leq 2 j<n \quad\left\langle P_{\mathfrak{A}}(S \circ T), \rho^{j}(P \star Q)\right\rangle= \\
& \sigma_{P} \sigma_{Q} \sum_{R} \frac{-\omega_{R}^{j}}{W_{R}}\left\{\overline{\sigma_{R}}\left(\sigma_{T} \bar{\sigma}_{S} \bar{\sigma}_{Q} \sigma_{P} \overline{b_{R}^{S T}} a_{R}^{P Q}+\overline{a_{R}^{S T}} b_{R}^{P Q}\right)\left(\omega_{R}[2(n-j)+1]+[2 j+1]\right)\right. \\
&\left.\quad+(-1)^{n+1}\left(\overline{\sigma_{P}} \sigma_{Q} \overline{a_{R}^{S T}} a_{R}^{P Q}+\sigma_{S} \overline{\sigma_{T}} \overline{b_{R}^{S T}} b_{R}^{P Q}\right)\left(\omega_{R}[n-2 j]+[n+2 j+2]\right)\right\}
\end{aligned}
$$

Proof. Note first that if $\{w\}$ is a basis for a finite dimensional Hilbert space with dual basis $\{\hat{w}\}$ then $\langle\hat{u}, \hat{v}\rangle$ is just the coefficient of $u$ in the expression of $\hat{v}$ in the basis $\{w\}$.

Let us show how the formulae are applied to obtain one of the coefficients. We want to calculate

$$
\left\langle\sum_{R \in \mathfrak{B}} \sigma_{R}^{n} a_{R}^{S T} \cup_{n+1} R+\sigma_{T}^{-1} \sigma_{S} b_{R}^{S T} \hat{\cup_{0}} R, \sum_{R \in \mathfrak{B}} \sigma_{R}^{n} a_{R}^{P Q} \cup_{n+1+2 j} R+\sigma_{Q}^{-1} \sigma_{P} b_{R}^{P Q} \cup_{2 j} R\right\rangle
$$

Obviously the inner products of terms with different $R$ 's are zero so the contribution of the first "cross-term" in the inner products is:

$$
\sum_{R \in \mathfrak{B}} \sigma_{R}^{-n} \sigma_{Q}^{-1} \sigma_{P} \overline{a_{R}^{S T}} b_{R}^{P Q}\left\langle\hat{\cup_{n+1}} \hat{R}, \hat{\cup_{2 j}} R\right\rangle
$$

but $\left\langle\cup_{n+1} R, \hat{\cup_{2 j}} R\right\rangle=\left\langle\cup_{n+1-2 j} R, \hat{\cup_{0}} R\right\rangle$ and $1 \leq n+1-2 j \leq n+1$ so by (ii) of 4.2 .9 it is equal to

$$
\begin{aligned}
& \frac{1}{W}\left\{\left(-\sigma_{R}\right)^{2 j-n-1}\left(\omega_{R}[n+1-2 j]+[n+1+2 j]\right)\right\}= \\
& \sigma_{R}^{n} \frac{(-1)^{n+1}}{W_{R}}\left\{\omega_{R}^{j} \sigma_{R}\left(\omega_{R}^{-1}[n+2 j+1]+[n-2 j+1]\right)\right\}
\end{aligned}
$$

and the other "cross-term" is

$$
\sum_{R \in \mathfrak{B}} \sigma_{R}^{-n} \sigma_{T} \sigma_{S}^{-1} \overline{b_{R}^{S T}} a_{R}^{P Q}\left\langle\hat{\cup_{0}} R, \cup_{n+1+2 j} R\right\rangle
$$

again $\left\langle\hat{\cup_{0}} R, \cup_{n+1+2 j} R\right\rangle=\left\langle\cup_{n+1-2 j} R, \hat{\cup_{0} R}\right\rangle$ so that the cross terms contribute the right amount to the formula.

The other terms are calculated in the same way.

Note that there is a nice check on these formulae.

When $n$ is even, say $n=2 k$, a picture shows that $\rho^{k}(P \circ Q)^{*}=\omega_{P}^{k-1} \omega_{Q}^{k} P \star Q$ so that

$$
\left\langle S \circ T, \rho^{k}(P \circ Q)\right\rangle=\omega_{P}^{k+1} \omega_{Q}^{k}\left\langle S \circ T,(P \star Q)^{*}\right\rangle
$$

In general $\left\langle X, Y^{*}\right\rangle=\overline{\left\langle X^{*}, Y\right\rangle}$ and since $(S \circ T)^{*}=T \circ S$ we have

$$
\left\langle S \circ T, \rho^{k}(P \circ Q)\right\rangle=\omega_{P}^{k+1} \omega_{Q}^{k} \overline{\langle T \circ S, P \star Q\rangle}
$$

If we calculate the left and right hand sides of this equation using the first and second parts of 4.4 .2 we see they agree using $\operatorname{Tr}(R P Q)=\left(\sigma_{R} \sigma_{P} \sigma_{Q}\right)^{n} \operatorname{Tr}(R Q P)=\overline{\operatorname{Tr}(R Q P)}$. 


\subsection{Projection onto TL}

Definition 4.5.1. Let $\mathbb{T}$ be the linear span of all TL diagrams in $P_{n+1}$.

It is obvious that $\mathfrak{A}$ and $\mathfrak{T}$ are orthogonal. To understand the following formulae note that $T L$ has a meaning as an unshaded planar algebra. This means we can interpret $\mathcal{F} x$ as an element in $T L_{k}$ for $x \in T L_{k}$ simply by reversing the shadings. We will use this convention frequently below.

\section{Proposition 4.5.2.}

$$
\begin{gathered}
P_{\mathfrak{T}}(S \circ T)=\left\{\begin{array}{cc}
\frac{p_{n+1}}{[n+2]} & \text { if } S=T \\
0 & \text { otherwise }
\end{array}\right. \\
P_{\mathfrak{T}}(S \star T)=\left\{\begin{array}{cc}
\frac{\omega_{S} \mathcal{F} p_{n+1}}{[n+2]} & \text { if } S=T \\
0 & \text { otherwise }
\end{array}\right. \\
P_{\mathfrak{T}}\left(\mathcal{F}^{j}(S \circ T)\right)=\mathcal{F}^{j}\left(P_{\mathfrak{T}}(S \circ T)\right)
\end{gathered}
$$

Proof. $P_{\mathfrak{T}}(S \circ T)$ is a multiple of $p_{n+1}$ by 2.6.5. Taking the trace gives the multiple. And $P \mathfrak{T}$ commutes with $\rho$.

We see that we will need the inner products of the JW idempotents with their rotated versions.

Lemma 4.5.3. For $m \geq i$,

$$
\left\langle p_{m}, \mathcal{F}^{i}\left(p_{m}\right)\right\rangle=(-1)^{m i}[m+1] \frac{[m-i][m-i-1] \ldots[1]}{[m][m-1] \ldots[i+1]}=(-1)^{i(m-i)} \frac{[m+1]}{\left[\begin{array}{c}
m \\
i
\end{array}\right]}
$$

Proof. Note the special cases $i=m, i=0$ which certainly work. So suppose $1 \leq i<m$. We will use Wenzl's inductive formula for $p_{m}: p_{m+1}=p_{m}-\frac{[m]}{[m+1]} p_{m} E_{m} p_{m}$. Drawing a picture for the inner product we see that the first term does not contribute by 2.6.5. The trace of the following picture represents $\left\langle p_{m+1}, p_{m} E_{m} p_{m}\right\rangle$ 


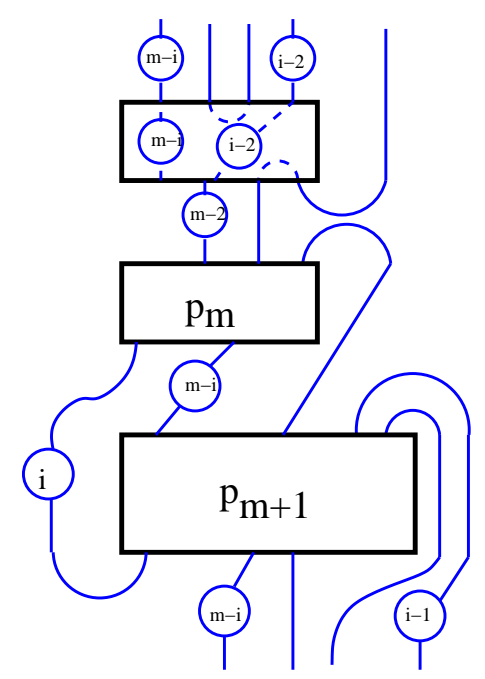

where we have used numbers in circles to indicate multiple strings. Inside the top box is the only TL element which makes a non-zero contribution. Its coefficient is $(-1)^{i} \frac{[m-i+1]}{[m]}$ by 2.6.7. Removing that box and doing some isotopy one gets the trace of the following picture:

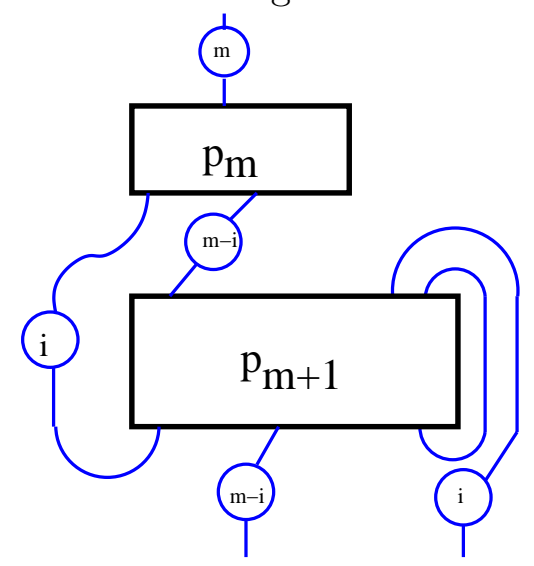

By formula 3 of 2.6.6 this is just $\frac{[m+2]}{[m+1]}\left\langle p_{m}, \mathcal{F}^{i}\left(p_{m}\right)\right\rangle$ and we end up with

$$
\left\langle p_{m+1}, \mathcal{F}^{i}\left(p_{m+1}\right)\right\rangle=(-1)^{i} \frac{[m-i+1][m+2]}{[m+1]^{2}}\left\langle p_{m}, \mathcal{F}^{i}\left(p_{m}\right)\right\rangle
$$

we may continue to apply the procedure. The last time we can do it, " $m+1$ " will be $i+1$ and the equation will be:

$$
\left\langle p_{i+1}, \mathcal{F}^{i}\left(p_{i+1}\right)\right\rangle=(-1)^{i} \frac{[1][i+2]}{[i+1]^{2}}\left\langle p_{i}, \mathcal{F}^{i}\left(p_{i}\right)\right\rangle
$$

After making all the cancellations in the product and observing that $\left\langle p_{i}, \mathcal{F}^{i}\left(p_{i}\right)\right\rangle=[i+1]$ we get the answer. 


\section{Applications to subfactors.}

\subsection{Multiplicity one.}

Let $\mathcal{P}$ be an (n-1)-supertransitive planar algebra with $n$-multiplicity one and chirality $\omega$.

We shall now make a careful choice of the elements $R$ (and $\check{R}$ ) that form the basis $\mathfrak{B}$ in section 4 So as in 3.5 .3 choose minimal central projections of $P_{n}$ with

$$
e+f=p_{n}
$$

$p_{n}$ being the JW projection (2.6.5). To be as precise as possible, suppose $\operatorname{Tr}(e) \leq \operatorname{Tr}(f)$ so that

5.1.1.

$$
r=\frac{\operatorname{Tr}(f)}{\operatorname{Tr}(e)} \geq 1
$$

Define $\tilde{R}$ orthogonal to $T L$ is

$$
\tilde{R}=r e-f .
$$

Since $e f=0$, algebra is easy and we obtain

5.1.3.

$$
\tilde{R}^{k}=r^{k} e+(-1)^{k} f
$$

so that

5.1 .4 .

$$
\tilde{R}^{2}=(r-1) \tilde{R}+r p_{n}
$$

5.1 .5 .

$$
\operatorname{Tr}\left(\tilde{R}^{2}\right)=r[n+1] \text {, }
$$

Definition 5.1.6. Set $R=\frac{\tilde{R}}{\sqrt{r[n+1]}}$

We have $\langle R, R\rangle=1$ and

5.1.7.

$$
\operatorname{Tr}\left(R^{3}\right)=\frac{1}{(r[n+1])^{3 / 2}} \operatorname{Tr}\left(r^{3} e-f\right)=\frac{\sqrt{r}-\frac{1}{\sqrt{r}}}{\sqrt{[n+1]}},
$$

Note that if $r \neq 1$ we have been able to choose $R$ without any ambiguity in sign. See 4.1 .1

We will also need formulae involving $\mathcal{E}$ of 2.6.3.

By multiplying $\mathcal{E}\left(R^{2}\right)$ and $\mathcal{E}\left(p_{n}\right)$ by the $E_{i}$ 's of 2.6.4 we see that they are both multiples of $p_{n-1}$ and on taking the trace we get

5.1.8. $\mathcal{E}\left(R^{2}\right)=\frac{p_{n-1}}{[n]}$ 
so that

5.1 .9 .

$$
\operatorname{Tr}\left(\mathcal{E}\left(R^{2}\right)^{2}\right)=\frac{1}{[n]}
$$

To be even-handed we need to do the same for $P_{n,-}$ so we will continue the convention of using a ${ }^{\sim}$ symbol to indicate the corresponding objects for $P_{n,-}$. Thus we have $\check{e}$ and $\check{f}$ and

5.1.10.

$$
\check{r}=\frac{\operatorname{Tr}(\check{f})}{\operatorname{Tr}(\check{e})} \geq 1
$$

and all the above formulae have $\checkmark$ versions. At this stage we have two potential meanings for $\check{R}$. To make them consistent just forces the choice of square root $\sigma$ of $\omega$ so that $\mathcal{F}(R)=$ $\sigma \check{R}$.

Note that if $r$ and $\check{r}$ are both different from 1 (i.e. neither $\operatorname{Tr}\left(R^{3}\right)$ nor $\operatorname{Tr}\left(\check{R}^{3}\right)$ is zero), there is no choice of signs anywhere - they are imposed by our conventions. We will use $\omega^{1 / 2}$ instead of $\sigma$.

Theorem 5.1.11. Let $\mathcal{P}$ be an $n-1$-supertransitive subfactor planar algebra with multiplicity sequence $0^{n-1} 10$ and chirality $\omega$. Let $r, \check{r}$ be as above. We may suppose $r \leq \check{r}$ (by passing to the dual if necessary). Then $n$ is even,

$$
\check{r}=\frac{[n+2]}{[n]}
$$

and

$$
r+\frac{1}{r}=2+\frac{2+\omega+\omega^{-1}}{[n][n+2]}
$$

If $\omega=-1, n$ is divisible by 4 .

Proof. Since the $(n+1)$-multiplicity is zero, $S \circ T, S \star T$ and their rotations are in the linear span of $\mathfrak{A}$ and $T L$ so we can calculate inner products between them using the formulae of the previous section. We record the ones we will use (with $W=W_{2 n+2, \omega}$ ):

Let $\alpha=\operatorname{Tr}\left(R^{3}\right)$ and $\operatorname{Tr}\left(\check{R}^{3}\right)=\beta$.

$$
\left\langle P_{\mathfrak{A}}(R \circ R), R \circ R\right\rangle=\frac{1}{W}\left(\left(\alpha^{2}+\beta^{2}\right)[2 n+2]+(-1)^{n+1} 2 \alpha \beta\left(\omega^{1 / 2}+\omega^{-1 / 2}\right)[n+1]\right)
$$

$\left\langle P_{\mathfrak{A}}(R \circ R), R \star R\right\rangle=$

$$
\frac{1}{W}\left\{(-1)^{n} \omega\left(\alpha^{2}+\beta^{2}\right)(\omega[n]+[n+2])-2 \alpha \beta \omega^{1 / 2}(\omega[2 n+1]+1)\right\}
$$

These are just 4.4 .2 with $j=0$ and $P=Q=S=T=R$. 
When $n$ is odd the second formula is just 4.4 .2 with $j=0$. If $n$ is even one may obtain it from the first formula of 4.4 .2 with $j=k$ by noting that $R \star R=\omega \rho^{k}(R \circ R)^{*}$.

We have, from 4.5.3 and 4.5.2 that

$$
\left\langle P_{\mathbb{T}}(R \circ R), R \circ R\right\rangle=\frac{1}{[n+2]}
$$

and

$$
\langle P \mathfrak{T}(R \circ R), R \star R\rangle=\frac{(-1)^{n} \omega}{[n+2][n+1]}
$$

But we can calculate $\langle R \circ R, R \circ R\rangle$ and $\langle R \circ R, R \star R\rangle$ directly from their pictures and the algebraic relations satisfied by $R$. The first is trivial-

$$
\langle R \circ R, R \circ R\rangle=\operatorname{Tr}\left(\mathcal{E}\left(R^{2}\right)^{2}\right)=\frac{1}{[n]}
$$

5.1.9. This implies the same value for $\langle R \star R, R \star R\rangle$. For $\langle R \circ R, R \star R\rangle$, a little isotopy produces the trace of the following picture:

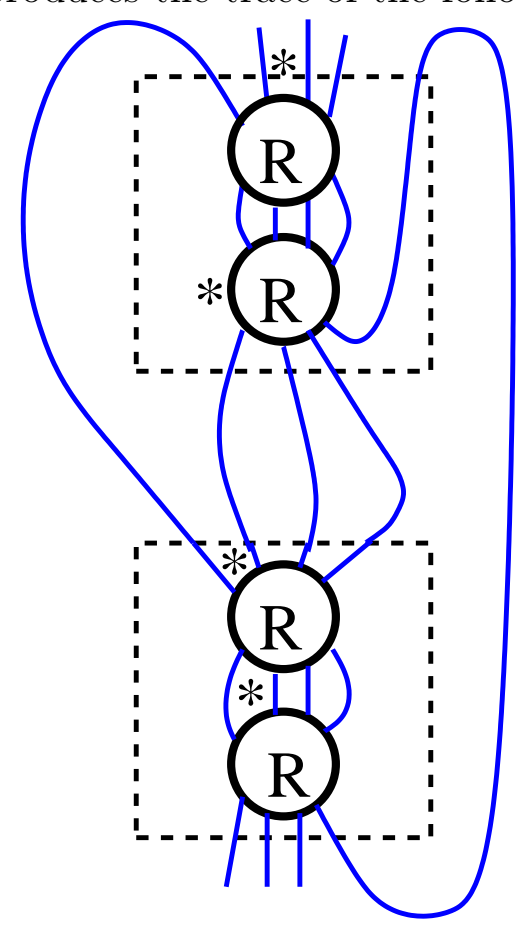

which we recognise as $\omega\left\langle\mathcal{F}\left(R^{2}\right), \mathcal{F}(R)^{2}\right\rangle=\omega^{2}\left\langle\mathcal{F}\left(R^{2}\right),(\check{R})^{2}\right\rangle$. And $R^{2}=\alpha R+\frac{p_{n}}{[n+1]},(\check{R})^{2}=$ $\beta \check{R}+\frac{p_{n}}{[n+1]}$ so we get $\omega^{-1 / 2} \alpha \beta+\frac{\left\langle\mathcal{F}\left(p_{n}\right), p_{n}\right\rangle}{[n+1]^{2}}$ so finally

$$
\langle R \circ R, R \star R\rangle=\omega^{3 / 2} \alpha \beta+\frac{(-1)^{n-1} \omega^{2}}{[n+1][n]}
$$


Now we may use zero $n+1$-multiplicity $=0$, which implies

$\langle R \circ R, R \circ R\rangle=\left\langle P_{\mathfrak{A}}(R \circ R), R \circ R\right\rangle+\left\langle P_{\mathfrak{T}}(R \circ R), R \circ R\right\rangle$ and

$\langle R \circ R, R \star R\rangle=\left\langle P_{\mathfrak{A}}(R \circ R), R \star R\right\rangle+\left\langle P_{\mathfrak{C}}(R \circ R), R \star R\right\rangle$ to obtain

$$
\frac{1}{[n]}=\frac{1}{W}\left(\left(\alpha^{2}+\beta^{2}\right)[2 n+2]+(-1)^{n+1} 2 \alpha \beta\left(\omega^{1 / 2}+\omega^{-1 / 2}\right)[n+1]\right)+\frac{1}{[n+2]}
$$

and $\omega^{3 / 2} \alpha \beta+\frac{(-1)^{n-1} \omega^{2}}{[n+1][n]}=$

$$
\frac{1}{W}\left\{(-1)^{n} \omega\left(\alpha^{2}+\beta^{2}\right)(\omega[n]+[n+2])-2 \alpha \beta \omega^{1 / 2}(\omega[2 n+1]+1)\right\}+\frac{(-1)^{n} \omega}{[n+2][n+1]}
$$

Using $\frac{1}{[n]}-\frac{1}{[n+2]}=\frac{[2 n+2]}{[n][n+1][n+2]}$ the first equation becomes

5.1.12.

$$
\frac{W}{[n][n+1][n+2]}=(-1)^{n+1} \frac{2 \alpha \beta\left(\omega^{1 / 2}+\omega^{-1 / 2}\right)}{q^{n+1}+q^{-n-1}}+\alpha^{2}+\beta^{2}
$$

and after a little work the second equation becomes:

\subsubsection{3.}

$$
\frac{W([n]+[n+2] \omega)}{[n][n+1][n+2]}=(-1)^{n} \alpha \beta \omega^{1 / 2}\left([2 n+2] \delta+\omega^{-1}-\omega\right)-\left(\alpha^{2}+\beta^{2}\right)([n] \omega+[n+2])
$$

Thus we have two linear equations for $\alpha^{2}+\beta^{2}$ and $\alpha \beta$. It is easy to check that they are satisfied by

$$
\begin{gathered}
\alpha \beta=\frac{(-1)^{n}\left(\omega^{1 / 2}+\omega^{-1 / 2}\right)\left(q^{n+1}+q^{-n-1}\right)}{[n][n+1][n+2]} \\
\alpha^{2}+\beta^{2}=\frac{\left(q^{n+1}+q^{-n-1}\right)^{2}+\left(\omega^{1 / 2}+\omega^{-1 / 2}\right)^{2}}{[n][n+1][n+2]}
\end{gathered}
$$

Now if $n$ is odd, there is a trace-preserving isomorphism between $P_{n,+}$ and $P_{n,-}$ given by a suitable power of the Fourier transform. Thus $\alpha=\beta$ which with these equations gives $q^{n+1}+q^{-n-1}+(-1)^{n+1}\left(\omega^{\frac{1}{2}}+\omega^{-\frac{1}{2}}\right)=0$ which is impossible if $\delta>2$. So $n$ is even.

It then follows that

$$
(\alpha \pm \beta)^{2}=\frac{\left(q^{n+1}+q^{-n-1} \pm\left(\omega^{\frac{1}{2}}+\omega^{-\frac{1}{2}}\right)\right)^{2}}{[n][n+1][n+2]}
$$

so that if $\alpha \leq \beta$ (which is the same as $r \leq \check{r}$ ), we get

$$
\beta=\frac{q^{n+1}+q^{-n-1}}{\sqrt{[n][n+1][n+2]}} \quad \text { and } \quad \alpha=\frac{\omega^{\frac{1}{2}}+\omega^{-\frac{1}{2}}}{\sqrt{[n][n+1][n+2]}} .
$$


These immediately imply the desired formulae.

To see that $n$ is divisible by 4 when $\omega=-1$, observe that 5.1 .12 implies that at least one of $\alpha$ and $\beta$ is non-zero, wolog suppose it's $\alpha$. Then $r \neq 1$ so the traces of $e$ and $f$ are different. But $\rho^{n / 2}$ acts as a trace-preserving automorphism on the linear span of $e$ and $f$ and so must be the identity. This forces $n / 2$ to be even.

It is more than a little sensible to check these formulae in examples where all the parameters are known. In the Fuss Catalan example with $a=\sqrt{2}$ we have, from 3.5.10 that

$$
n=2, \delta=q+q^{-1}=b \sqrt{2}, \check{r}=2\left(b^{2}-1\right), r=\frac{b^{2}}{b^{2}-1} \text { and } \omega=1
$$

and the formulae of the theorem are rapidly verified. For the partition planar algebra

$$
n=4, \check{r}=\frac{\delta^{4}-4 \delta^{2}+3}{\delta^{2}-2}, r=\frac{\delta^{4}-3 \delta^{2}+2}{\delta^{4}-3 \delta^{2}} \text { and } \omega=1 .
$$

Corollary 5.1.14. The principal graph (corresponding to $\check{r}$ ) is as below for vertices of distance $\leq n+2$ from *:

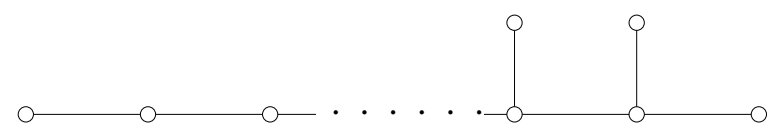

The other principal graph is as below:

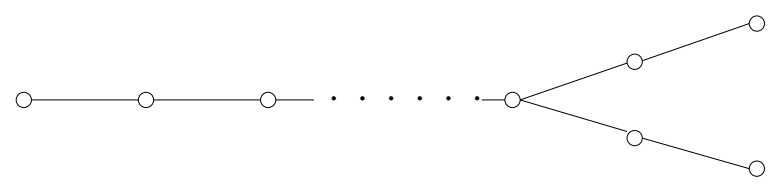

Proof. By the dimension constraints the principal graph for $\check{r}$ must begin as one of the two pictures. But the equation

$$
\check{r}=\frac{[n+2]}{[n]}
$$

is just the Perron Frobenius eigenvector equation for the first graph (at the top univalent vertex). So the eigenvalue equation of the second graph cannot satisfy that equation.

Corollary 5.1.15. The Haagerup planar algebra has chirality -1, but the Haagerup-Asaeda planar algebra has chirality 1.

Proof. As soon as one of the principal graphs has a symmetry exchanging the two vertices at distance $n$ from ${ }^{*}$, one has that $r=1$. This is the case for the Haagerup subfactor. The Asaeda-Haagerup value can be deduced from the Perron Frobenius data. 


\subsection{Multiplicity two.}

Begin by choosing an orthonormal basis $\mathfrak{B}=\{S, T\}$ of self-adjoint rotational eigenvectors for the orthogonal complement of $T L_{n}$ in $P_{n}$, with eigenvalues $\omega_{S}$ and $\omega_{T}$. And the corresponding $\sigma_{S}, \sigma_{T}, \check{S}$ and $\check{T}$.

Recall the notation $a_{R}^{P Q}=\operatorname{Tr}(R P Q)$. The ideal $\mathfrak{I}$ in $P_{n}$ which is the orthogonal complement of the TL elements with less than $n$ through strings is orthogonally spanned by $S, T$ and $p_{n}$. It is a commutative $C^{*}$-algebra whose identity is $p_{n}$. By taking inner products the multiplication law for $\mathfrak{I}$ is:

$$
\begin{aligned}
S^{2} & =a_{S}^{S S} S+a_{S}^{S T} T+\frac{p_{n}}{[n+1]} \\
T^{2} & =a_{S}^{T T} S+a_{T}^{T T} T+\frac{p_{n}}{[n+1]} \\
S T & =a_{S}^{S T} S+a_{S}^{T T} T
\end{aligned}
$$

Proposition 5.2.1. Associativity constraint.

$$
\left(a_{S}^{S T}\right)^{2}+\left(a_{S}^{T T}\right)^{2}=a_{S}^{S S} a_{S}^{T T}+a_{T}^{T T} a_{S}^{S T}+\frac{1}{[n+1]}
$$

Proof. Write $x=a_{S}^{S S}, y=a_{T}^{T} T$ and $u=a_{S}^{S T}, v=a_{S}^{T T}$. Evaluate $S^{2} T$ in two ways:

(a) $\left(S^{2}\right) T=\left(x S+u T+\frac{p_{n}}{[n+1]}\right) T=(x u+u v) S+\left(x v+u y+\frac{1}{[n+1]}\right) T+u \frac{p_{n}}{[n+1]}$

(b) $S(S T)=S(u S+v T)=(u x+v u) S+\left(u^{2}+v^{2}\right) T+u \frac{p_{n}}{[n+1]}$. The conclusion follows immediately.

Theorem 5.2.2. For $k \in \mathbb{Z}, k>0$, there is no 2k-supertransitive subfactor with multiplicity sequence beginning *20.

Proof. We will actually show that any such subfactor has to have index $\geq 4.5$ which is enough by Haagerup's classification ([8]), or as Snyder has pointed out, the smallest graph which could have multiplicity sequence beginning $* 20$ has norm-squared equal to $\frac{5+\sqrt{17}}{2}$ which is bigger than 4.5 .

Let $n=2 k+1$. The rotation $\mathcal{F}^{n}$ gives a trace preserving antiisomorphism between $P_{n,+}$ and $P_{n,-}$. And a picture shows immediately that if $R, P, Q \in\{S, T\}$,

$$
\operatorname{Tr}(\check{R} \check{P} \check{Q})=\left(\sigma_{R} \sigma_{P} \sigma_{Q}\right)^{n} \operatorname{Tr}(R Q P) .
$$

Moreover $S$ and $T$ all commute since the multiplicity is only 2 and $S$ and $T$ are commuting and self-adjoint which shows that $\operatorname{Tr}(R P Q), \operatorname{Tr}(\check{R} \check{P} \check{Q}) \in \mathbb{R}$ so $\operatorname{Tr}(R P Q)= \pm \operatorname{Tr}(\check{R} \check{P} \check{Q})$. We specialise the master formula 4.4 .2 to the case $P=T, Q=S, j=0$ which gives us $\left\langle P_{\mathfrak{A}}(S \circ T), T \circ S\right\rangle$. By 4.5 .2 and the multiplicity sequence beginning $* 20$, this is the same as $\langle S \circ T, T \circ S\rangle$ which is clearly zero. We are summing over the two values $S$ and $T$ of $R$. Note that, using the conventions of 4.4 .2 we have 


$$
\begin{array}{ll}
a_{S}^{S T}=a_{S}^{P Q}=\operatorname{Tr}\left(S^{2} T\right), \text { call it } a_{S}, & b_{S}^{S T}=b_{S}^{P Q}= \pm a_{S} \\
a_{T}^{S T}=a_{T}^{P Q}=\operatorname{Tr}\left(S T^{2}\right), \text { call it } a_{T}, & b_{T}^{S T}=b_{T}^{P Q}= \pm a_{T}
\end{array}
$$

Since $S$ and $T$ are self-adjoint and commute, $a_{S}$ and $a_{T}$ are real and we obtain

$$
\begin{aligned}
0= & \frac{a_{S}^{2}}{W_{S}}\left([2 n+2]\left(1+\omega_{S}^{-1} \omega_{T}\right)+\alpha[n+1]\right) \\
& +\frac{a_{T}^{2}}{W_{T}}\left([2 n+2]\left(1+\omega_{S}^{-1} \omega_{T}\right)+\beta[n+1]\right)
\end{aligned}
$$

Where $\alpha$ and $\beta$ are sums of four roots of unity. Multiplying through by a square root of $\omega_{S} \omega_{T}^{-1}$ which is also an $n$ th. root of unity we get

$$
0=\left(2 \cos \frac{2 r \pi}{n}[2 n+2]+\alpha^{\prime}[n+1]\right) \frac{a_{S}^{2}}{W_{S}}+\left(2 \cos \frac{2 r \pi}{n}[2 n+2]+\beta^{\prime}[n+1]\right) \frac{a_{T}^{2}}{W_{T}}
$$

for some integer $r$, where $\left|\alpha^{\prime}\right|$ and $\left|\beta^{\prime}\right|$ are $\leq 4$. We want to show that the $[2 n+2]$ term dominates so that the real parts of the coefficients of $a_{S}^{2}$ and $a_{T}^{2}$ get their sign from the $2 \cos \frac{2 r \pi}{n}[2 n+2]$. For this it clearly suffices to show that

$$
\left|2 \cos \frac{2 r \pi}{n}[2 n+2]\right|>4[n+1] .
$$

We again use that $n$ is odd-the smallest $\left|2 \cos \frac{2 r \pi}{n}\right|$ can be is $2 \sin \pi / 2 n$ and the inequality becomes:

$$
[2 n+2]>\frac{2[n+1]}{\sin \pi / 2 n} .
$$

Writing $s$ for $\frac{1}{\sin \pi / 2 n}$ and $Q$ for $q^{n+1}$ this becomes $Q^{2}-Q^{-2}>2 s\left(Q-Q^{-1}\right)$. Completing the square and using $Q>1$ we see that this is implied by $Q>2 s$. But $(\pi / 2 n) s$ decreases to 1 as $n$ increases so $2 s<\frac{2 \pi / 6}{\sin \pi / 6} \frac{2 n}{\pi}$. Hence it suffices to prove $q^{n+1} \geq \frac{4 n}{3}$. Consider the graphs of $q^{x+1}$ and (4/3)x. Clearly the $x$ coordinate of the largest point of intersection is decreasing as a function of $q$. So if we can show that this value of $x$ is 3 for $q=\sqrt{2}$ then for all greater $q$ and all $n \geq 3$ we will have $q^{n+1}>\frac{4 n}{3}$. But $(\sqrt{2})^{3+1}=4$ and by calculus $q^{x+1}-\frac{4 x}{3}$ is (just) increasing for $x=3$.

The value $q=\sqrt{2}$ corresponds to index 4.5. Since $q>1$ the $W$ factors are positive so we can conclude that, for index $\geq 4.5, a_{S}=a_{T}=0$. This contradicts the associativity constraint.

Notes.

(i) There is nothing special about 4.5, it was chosen simply because it suffices and the estimates are convenient.

(ii) The even supertransitivity assumption is necessary since the GHJ subfactor of index $3+\sqrt{3}([5,[4])$ has multiplicity sequence beginning $* 20$.

We end with an intriguing observation about chirality in the $* 20$ case. 
Theorem 5.2.3. Let $N \subset M$ be a subfactor with multiplicity sequence beginning $* 20$, and let $S, T, \omega_{S}$ and $\omega_{T}$ be as above. Then $\omega_{S} \neq \omega_{T}$.

Proof. Consider (i) of 4.4.1. As we have observed, $S$ and $T$ commute and have zero projection onto TL. Thus if $\sigma_{S}^{-1} \sigma_{T}=\sigma_{T}^{-1} \sigma_{S}$ we have $S \circ T=T \circ S$ which is impossible since $S \circ T$ and $T \circ S$ are orthogonal and non-zero.

\section{References}

[1] Asaeda, M. and Haagerup, U. (1999). Exotic subfactors of finite depth with Jones indices $(5+\sqrt{13}) / 2$ and $(5+\sqrt{17}) / 2$. Communications in Mathematical Physics, 202, $1-63$.

[2] Bigelow, S., Morrison, S., Peters, E. and Snyder, N. Constructing the extended Haagerup planar algebra. arXiv:0909.4099

[3] Bisch, D. and Jones, V. F. R. (1997). Algebras associated to intermediate subfactors. Inventiones Mathematicae, 128, 89-157.

[4] Evans, D. E. and Kawahigashi, Y. (1998). Quantum symmetries on operator algebras. Oxford University Press.

[5] Goodman, F., de la Harpe, P. and Jones, V. F. R. (1989). Coxeter graphs and towers of algebras. MSRI Publications (Springer), 14.

[6] Graham, J.J. and Lehrer, G.I. (1998) The representation theory of affine Temperley Lieb algebras. L'Enseignement Mathématique 44,1-44.

[7] Grossman, P., and Jones, V.F.R.(2007) Intermediate subfactors with no extra structure. J. Amer. Math. Soc. 20 , no. 1, 219-265.

[8] Haagerup, U. (1994). Principal graphs of subfactors in the index range $4<3+\sqrt{2}$. in Subfactors - Proceedings of the Taniguchi Symposium, Katata -, (ed. H. Araki, et al.), World Scientific, 1-38.

[9] Halverson, T. and Ram, A.(2005) Partition algebras. European Journal of Combinatorics. 26 869-921.

[10] Jones, V.F.R. (1994) An affine Hecke algebra quotient in the Brauer Algebra. l'Enseignement Mathematique 40, 313-344.

[11] Jones, V.F.R. and Reznikoff, S. (2006) Hilbert Space representations of the annular Temperley-Lieb algebra. Pacific Math Journal 228, 219-250

[12] Jones, V. F. R. (1983). Index for subfactors. Inventiones Mathematicae, 72, 1-25. 
[13] Jones, V. F. R. (in press). Planar algebras I. New Zealand Journal of Mathematics. QA $/ 9909027$

[14] Jones, V. F. R. (2001). The annular structure of subfactors. in Essays on geometry and related topics, Monographies de L'Enseignement Mathematique, 38, 401-463.

[15] Jones, V. F. R. (1994). The Potts model and the symmetric group. in Subfactors Proceedings of the Taniguchi Symposium, Katata -, (ed. H. Araki, et al.), World Scientific, 259-267.

[16] Jones, V. F. R. (2003) Quadratic tangles in planar algebras. In preparation: http://math.berkeley.edu/ vfr/

[17] Martin,P.P. (2000) The partition algebra and the Potts model transfer matrix spectrum in high dimensions. J.Phys. A 32, 3669-3695.

[18] Peters, E. (2009) A planar algebra construction of the Haagerup subfactor. arXiv:0902.1294

[19] Popa, S. (1990). Classification of subfactors: reduction to commuting squares. Inventiones Mathematicae, 101, 19-43.

[20] Popa, S. (1995). An axiomatization of the lattice of higher relative commutants of a subfactor. Inventiones Mathematicae, 120, 427-446.

[21] Temperley, H. N. V. and Lieb. E. H. (1971). Relations between the "percolation" and "colouring" problem and other graph-theoretical problems associated with regular planar lattices: some exact results for the "percolation" problem. Proceedings of the Royal Society A, 322, 251-280.

[22] Wenzl, H. (1987). On sequences of projections. Comptes Rendus Mathématiques, La Société Royale du Canada, L'Academie des Sciences, 9, 5-9. 